\title{
SQUARING IN CYCLOTOMIC SUBGROUPS
}

\author{
KORAY KARABINA
}

\begin{abstract}
We propose new squaring formulae for cyclotomic subgroups of the multiplicative group of certain finite fields. Our formulae use a compressed representation of elements having the property that decompression can be performed at a very low cost. The squaring formulae lead to new exponentiation algorithms in cyclotomic subgroups which outperform the fastest previouslyknown exponentiation algorithms when the exponent has low Hamming weight. Our algorithms can be adapted to accelerate the final exponentiation step of pairing computations.
\end{abstract}

\section{INTRODUCTION}

One challenge in cryptography is to achieve a desired level of security in the most efficient way. The efficiency can generally be improved if one can implement a cryptosystem with more compact parameters. An example in the context of discrete logarithm cryptosystems is the performance of 256-bit elliptic curve groups defined over 256-bit finite fields versus 256-bit subgroups of the multiplicative group of 3072-bit finite fields. Even though both systems are believed to provide 128-bit security with a careful choice of parameters, the former leads to a more efficient implementation than the latter mainly because the points in the corresponding elliptic curve group are represented with fewer bits than the elements in the subgroup of the multiplicative group of the corresponding finite field.

In recent years, there have been several proposals to represent the elements of cyclotomic subgroups of the multiplicative group of finite fields with fewer bits than is required in their natural representation and to compute with the compressed representation of elements [26, 9, 15, 18, 8, 22, 11, 30, 29, 25, 15, 16, 27, 14. These methods help close the gap between the efficiency of elliptic curve cryptosystems and finite field based cryptosystems. A related research objective is to improve the efficiency of finite field arithmetic using the special structure of cyclotomic subgroups. The most recent work is by Granger and Scott [12 who improved and extended the results in $[10$ and $[28$. They showed that if $q \equiv 1(\bmod 6)$, then the squaring operation in the order- $\left(q^{2}-q+1\right)$ cyclotomic subgroup of $\mathbb{F}_{q^{6}}^{*}$ can be performed at a cost of only 6 multiplications in $\mathbb{F}_{q}$ (or 3 squarings in $\mathbb{F}_{q^{2}}$ ).

We should emphasize that there are squaring algorithms that work with compressed representations of elements and that are faster than the method proposed in [12] for the order- $\left(q^{2}-q+1\right)$ cyclotomic subgroup $G$ of $\mathbb{F}_{q^{6}}^{*}$. For example, if $g \in G$, then one can adapt the XTR technique [18] to compute $\operatorname{Tr}_{\mathbb{F}_{q^{6}} / \mathbb{F}_{q^{2}}}\left(g^{2}\right)$ from

Received by the editor October 22, 2010 and, in revised form, August 22, 2011.

2010 Mathematics Subject Classification. Primary 94A60, 12E20, 14 G50.

Key words and phrases. Cyclotomic subgroups, squaring, exponentiation, pairing-based cryptography. 
$\operatorname{Tr}_{\mathbb{F}_{q^{6}} / \mathbb{F}_{q^{2}}}(g)$ at a cost dominated by 2 multiplications in $\mathbb{F}_{q}$. However, since the trace function is not multiplicative, cyptographic protocols that require multiplying more than two elements in $G$ do not seem to benefit from this fast trace-based squaring method. In particular, given only the traces of elements, the cost of recovering the full representation of these elements (decompression) and also the cost of multiplication with the trace representation greatly dominate the cost of multiplying two elements given in their natural representations in $\mathbb{F}_{q^{6}}$. In addition, even though the trace-based methods yield single-exponentiation and double-exponentiation algorithms that are faster than their conventional counterparts, it is not known how to use trace-based methods in general multi-exponentiation algorithms. Therefore, it is natural to look for squaring formulae that work with compressed representations and can be effectively incorporated into cryptographic applications.

In Section 2 we classify the known squaring techniques for cyclotomic subgroups in two categories: those that work with natural representation of elements, and those that work with compressed representation of elements. We will focus on the order $\left(q^{2}-q+1\right)$-cyclotomic subgroup $G$ of $\mathbb{F}_{q^{6}}^{*}$ as this seems to be the most interesting case with respect to some potential applications in pairing-based cryptography. In Section 3. we present a new formula for squaring elements in $G$ when $q \equiv 1(\bmod 6)$. We first describe a function $\mathcal{C}$ that compresses elements $g \in G$ by a factor of $3 / 2$, and describe a decompression function $\mathcal{D}$ that can be computed very efficiently and satisfies $\mathcal{D}(\mathcal{C}(g))=g$ for all $g \in G$. Our new squaring method works with this compressed form of elements. Given $\mathcal{C}(g)$, the cost of computing $\mathcal{C}\left(g^{2}\right)$ is dominated by 4 multiplications in $\mathbb{F}_{q}$ (or 2 squarings in $\mathbb{F}_{q^{2}}$ ). Note that this is $33 \%$ faster than the method described in [12]. The efficient decompression will permit us to effectively utilize the squaring formula in exponentiation algorithms, especially when the exponent has low Hamming weight. In Section 4, we discuss some applications of our squaring formula and provide some comparisons based on operation counts. In Section 5. we describe a more general technique to search for efficient squaring formulae. As a result, we discover other squaring formulae some of which seem to offer better performance in particular cases. Section 5 also shows that some of the previously-known squaring formulae can be obtained via our search method. In Section [6 we compare our new squaring formulae and the squaring formulae in [12. We conclude in Section 7.

\section{A REVIEW OF SQUARING AND EXPONENTIATION ALGORITHMS IN CYCLOTOMIC SUBGROUPS OF $\mathbb{F}_{q^{6}}^{*}$}

Let $\mathbb{F}_{q}$ be a finite field with $q$ elements and characteristic not equal to 2 or 3 . For simplicity, we first assume that $q=p$ is prime. We denote by $G_{\Phi_{6}(p)}$ the order- $\Phi_{6}(p)$ cyclotomic subgroup of $\mathbb{F}_{p^{6}}^{*}$. Here, $\Phi_{i}(p)$ denotes the $i$ th cyclotomic polynomial evaluated at $p$, and $\left|G_{s}\right|=s$. Note that $\left|G_{\Phi_{6}(p)}\right|=p^{2}-p+1$. Since $\mathbb{F}_{p^{6}}$ is the smallest extension of $\mathbb{F}_{p}$ that contains $G_{\Phi_{6}(p)}$, an element $g \in G_{\Phi_{6}(p)}$ is naturally represented with $6 \mathbb{F}_{p}$-elements. However, exploiting the algebraic structure of $G_{\Phi_{6}(p)}$, one can represent $g \in G_{\Phi_{6}(p)}$ with 3 or even with $2 \mathbb{F}_{p^{-}}$-elements yielding more compact representations by factor 2 or 3 . Trace-based compression and torus-based compression are the two known methods to achieve factor 2 and 3 compression in $G_{\Phi_{6}(p)}[26,18,22$. We summarize next the fastest previouslyknown squaring algorithms in $G_{\Phi_{6}(p)}$. These algorithms fall into two categories: 
those that work with compressed representation of elements, and those that work with full representation of elements.

\subsection{Compressed representations.}

2.1.1. Trace-based squaring: Let $\operatorname{Tr}_{p^{i}, p^{j}}$ denote the trace function $\operatorname{Tr}_{\mathbb{F}_{p^{i}} / \mathbb{F}_{p^{j}}}$ : $\mathbb{F}_{p^{i}} \rightarrow \mathbb{F}_{p^{j}}$. Elements $g \in G_{\Phi_{6}(p)}$ can be uniquely represented by their traces $\operatorname{Tr}_{p^{6}, p^{3}}(g)$ (up to conjugation over $\mathbb{F}_{p^{3}}$ ) [26], or $\operatorname{Tr}_{p^{6}, p^{2}}(g)$ (up to conjugation over $\mathbb{F}_{p^{2}}$ ) [18. More interestingly, one can compute $\operatorname{Tr}_{p^{6}, p^{3}}\left(g^{2}\right)$ and $\operatorname{Tr}_{p^{6}, p^{2}}\left(g^{2}\right)$ given $\operatorname{Tr}_{p^{6}, p^{3}}(g)$ and $\operatorname{Tr}_{p^{6}, p^{2}}(g)$, respectively. The corresponding squaring algorithms are known as LUC squaring and XTR squaring, respectively.

LUC-squaring: $\operatorname{Tr}_{p^{6}, p^{3}}\left(g^{2}\right)=\operatorname{Tr}_{p^{6}, p^{3}}(g)^{2}-2$. The cost of LUC-squaring is dominated by 1 squaring in $\mathbb{F}_{p^{3}}$.

XTR-squaring: $\operatorname{Tr}_{p^{6}, p^{2}}\left(g^{2}\right)=\operatorname{Tr}_{p^{6}, p^{2}}(g)^{2}-2 \operatorname{Tr}_{p^{6}, p^{2}}(g)^{p}$. The cost of XTR-squaring is dominated by 1 squaring in $\mathbb{F}_{p^{2}}$ (since the cost of the Frobenius $c \mapsto c^{p}$ is negligible for $c \in \mathbb{F}_{p^{2}}$ when using an optimal normal basis).

2.1.2. Torus-based squaring: Let $\mathbb{F}_{p^{6}}=\mathbb{F}_{p^{3}}(\sigma)$ where $\sigma$ is a root of $x^{2}-c$ for some quadratic non-residue $c \in \mathbb{F}_{p^{3}}$. Elements $g=g_{0}+g_{1} \sigma \in G_{\Phi_{6}(p)} \backslash\{ \pm 1\}$ can be uniquely represented by $\alpha=\left(g_{0}+1\right) / g_{1} \in \mathbb{F}_{p^{3}}$. In fact, if $\alpha \in \mathbb{F}_{p^{3}}$ is the compact representation of $g \in G_{\Phi_{6}(p)}$, then $g=(\alpha+\sigma) /(\alpha-\sigma)$ and $g^{2}=\left(\alpha^{2}+\right.$ $c+2 \alpha \sigma) /\left(\alpha^{2}+c-2 \alpha \sigma\right)$; see [22, 23]. Now, given $g^{2^{i}}=(x+y \sigma) /(x-y \sigma) \in G_{\Phi_{6}(p)}$ for some $x, y \in \mathbb{F}_{p^{3}}$, one can write $g^{2^{i+1}}=\left(x^{2}+y^{2} c+2 x y \sigma\right) /\left(x^{2}+y^{2} c-2 x y \sigma\right)$. In order to avoid the inversion operation in the squaring formula, one can encode an element $g=(x+y \sigma) /(x-y \sigma) \in G_{\Phi_{6}(p)} \backslash\{ \pm 1\}$ with $\mathcal{P}(g)=[x, y]$ and compute $\mathcal{P}\left(g^{2}\right)=\left[x^{2}+y^{2} c, 2 x y\right]$, the (unique) representative of $g^{2}$. This is the so-called squaring with mixed/projective coordinates, which was introduced in [11]. We call this the TB2-squaring method as it uses factor-2 torus-based compression.

TB2-squaring: $\mathcal{P}\left(g^{2}\right)=\left[x^{2}+y^{2} c, 2 x y\right]$, where $\mathcal{P}(g)=[x, y]$. The cost of TB2squaring is dominated by 2 multiplications in $\mathbb{F}_{p^{3}}$ because $x^{2}+y^{2} c=(x+y c)(x+$ $y)-(c+1) x y$ and we can ignore the cost of addition, subtraction, and multiplication by $c$.

Remark 2.1. Given $g=(\alpha+\sigma) /(\alpha-\sigma) \in G_{\Phi_{6}(p)}$ for some $\alpha \in \mathbb{F}_{p^{3}}$, one can exploit the algebraic structure of $G_{\Phi_{6}(p)}$ to further compress $\alpha$ to two $\mathbb{F}_{p}$-elements [22, 11. One can then define TB3-squaring analogous to TB2-squaring. However, to the author's knowledge, this extra structure has not yet been exploited to derive efficient TB3-squaring formulae. For example, it was reported in 11 that the cost of a TB3-squaring that uses the factor-3 torus-based compressed representation of elements in $G_{\Phi_{6}(p)}$ is 21 multiplications, 38 additions and 1 inversion in $\mathbb{F}_{p}$ (when $p \equiv 2,5(\bmod 9))$. Even though the inversion can be eliminated by using mixed/projective coordinates as in TB2-squaring, the cost is still dominated by 21 multiplications in $\mathbb{F}_{p}$, which is more expensive than the above TB2-squaring.

2.2. Full representations. The squaring formulae that work with the natural representation of $g$ can be summarized as below. 
General squaring: Let $\mathbb{F}_{p^{6}}=\mathbb{F}_{p^{3}}(\sigma)$, where $\sigma$ is a root of $\sigma^{2}-c$ for some quadratic non-residue in $\mathbb{F}_{p^{3}}$. Let $g=g_{0}+g_{1} \sigma \in \mathbb{F}_{p^{6}}^{*}$. Then $g^{2}=\left(g_{0}^{2}+g_{1}^{2} c\right)+2 g_{0} g_{1} \sigma$ can be computed at a cost dominated by 2 multiplications in $\mathbb{F}_{p^{3}}$ because $g_{0}^{2}+g_{1}^{2} c=$ $\left(g_{0}+g_{1} c\right)\left(g_{0}+g_{1}\right)-(c+1) g_{0} g_{1}$ and we may again ignore the cost of addition, subtraction, and multiplication by $c$.

SL-squaring: Let $p \equiv 2,5(\bmod 9)$ and $g \in G_{\Phi_{6}(p)} \subset \mathbb{F}_{p^{6}}^{*}$. Using the algebraic relations induced by $g^{p^{3}+1}=g^{p^{2}-p+1}=1$ on the coefficients of the vector representation of $g$ over $\mathbb{F}_{p}$, Stam and Lenstra 28] showed that $g^{2}$ can be computed at a cost dominated by 6 multiplications in $\mathbb{F}_{p}$. Moreover, when $p \equiv 2(\bmod 3)$ or $p \equiv 3(\bmod 4)$, the algebraic relations induced by $g^{p^{3}+1}=1$ on the coefficients of the vector representation of $g$ over $\mathbb{F}_{p^{3}}$ were used to compute $g^{2}$ at a cost dominated by 2 squarings in $\mathbb{F}_{p^{3}}$. We call the Stam-Lenstra methods SL1-squaring and SL2-squaring, respectively.

GPS-squaring: Let $p \equiv 1(\bmod 12)$ and $g \in G_{\Phi_{6}(p)} \subset \mathbb{F}_{p^{6}}^{*}$. Using the algebraic relations induced by $g^{p^{3}+1}=g^{p^{2}-p+1}=1$ on the coefficients of the vector representation of $g$ over $\mathbb{F}_{p}$, Granger, Page and Smart 10 showed that $g^{2}$ can be computed at a cost dominated by 3 multiplications and 6 squarings in $\mathbb{F}_{p}$.

GS-squaring: Let $q \equiv 1(\bmod 6)$ be a prime power and $g \in G_{\Phi_{6}(q)} \subset \mathbb{F}_{q^{6}}$. Granger and Scott [12, using the algebraic relations induced by $g^{q^{3}+1}=g^{q^{2}-q+1}=$ 1 on the coefficients of the vector representation of $g$ over $\mathbb{F}_{q^{2}}$, showed that $g^{2}$ can be computed at a cost dominated by 3 squarings in $\mathbb{F}_{q^{2}}$.

Table 1 summarizes the dominating costs of the above-mentioned squaring algorithms. We let $M_{i}$ and $S_{i}$ denote multiplication and squaring costs in $\mathbb{F}_{p^{i}}$. We may assume a) $M_{3 i}=6 M_{i}$ using Karatsuba's technique; b) $S_{2 i}=2 M_{i}$ using general squaring as above; and c) $S_{3 i}=M_{i}+4 S_{i}$ using Chung and Hasan's generalized Tom-Cook squaring formulae $\mathrm{SQR}_{3}[6]$.

TABLE 1. A summary of squaring algorithms in $G_{\Phi_{6}(p)}$.

\begin{tabular}{|l|l|l|}
\hline Algorithms & Cost & Restriction \\
\hline \hline Compressed representation \\
\hline LUC & $1 S_{3}=1 M_{1}+4 S_{1}$ & decompression \\
\hline XTR & $1 S_{2}=2 M_{1}$ & decompression \\
\hline TB2 & $2 M_{3}=12 M_{1}$ & \\
\hline Full representation \\
\hline General & $2 M_{3}=12 M_{1}$ & \\
\hline SL1 & $6 M_{1}$ & $p \equiv 2,5(\bmod 9)$ \\
\hline SL2 & $2 S_{3}=2 M_{1}+8 S_{1}$ & $p \equiv 2(\bmod 3), p \equiv 3(\bmod 4)$ \\
\hline GPS & $3 M_{1}+6 S_{1}$ & $p \equiv 1(\bmod 12)$ \\
\hline GS & $3 S_{2}=6 M_{1}$ & $p \equiv 1(\bmod 6)$ \\
\hline
\end{tabular}

We should note that the squaring algorithms described above for $G_{\Phi_{6}(p)}$ can be generalized to obtain squaring algorithms for $G_{\Phi_{6 i}(p)} \subset \mathbb{F}_{p^{6 i}}$ when all prime divisors of $i$ divide 6 . Indeed, one can replace $p$ by $q=p^{i}$ in the arguments and note that $G_{\Phi_{6 i}(p)}=G_{\Phi_{6}(q)} \subset \mathbb{F}_{q^{6}}^{*}[1]$ The dominating costs of the corresponding

\footnotetext{
${ }^{1}$ Under this generalization the GPS-squaring algorithm seems to scale better than the one described in [10] for $i=2,3$ and 4 .
} 
squaring algorithms may be obtained by replacing each $M_{j}$ and $S_{j}$ in Table 1 by $M_{j \cdot i}$ and $S_{j \cdot i}$, respectively (and the restrictions on $p$ in Table 1 should then be read as restrictions on $q$ ).

Even though XTR-squaring seems to be the fastest squaring algorithm for $G_{\Phi_{6 i}(p)}$ $\subset \mathbb{F}_{p^{6 i}}\left(\right.$ or, $G_{\Phi_{6}(q)} \subset \mathbb{F}_{q^{6}}^{*}$ with $\left.q=p^{i}\right)$ for $i \in\{1,2,3,4\}$, it suffers from the nonmultiplicative property of the trace function as mentioned in Section 1 . Consequently, GS-squaring and SL1-squaring seem to be the best squaring algorithms that can be easily deployed in cryptographic algorithms. In addition, GS-squaring has the extra advantage that it allows the use of pairing-friendly or toweringfriendly fields for more efficient implementation of pairing-based cryptographic protocols; see [17, 3].

\section{A NEW SQUARING FORMULA IN CYCLOTOMIC SUBGROUPS}

Let $q=p^{i} \equiv 1(\bmod 6)$ be a prime power and $G_{\Phi_{6}(q)} \subset \mathbb{F}_{q^{6}}^{*}$. We let $\mathbb{F}_{q^{2}}=\mathbb{F}_{q}(w)$ where $w^{2}=c, c \in \mathbb{F}_{q}$ is a quadratic non-residue, $w \in \mathbb{F}_{q^{2}}$ is a cubic non-residue; and $\mathbb{F}_{q^{6}}=\mathbb{F}_{q^{2}}(\sigma)$ where $\sigma^{3}=w$. Then it is easy to show that

$$
\begin{aligned}
w^{q} & =-w, \\
\sigma^{q} & =m \sigma,
\end{aligned}
$$

where $m \in \mathbb{F}_{q}$ is some primitive sixth root of unity.

If $g \in \mathbb{F}_{q^{6}}$, then we write

$$
g=\left(g_{0}+g_{1} w\right)+\left(g_{2}+g_{3} w\right) \sigma+\left(g_{4}+g_{5} w\right) \sigma^{2},
$$

where $g_{i} \in \mathbb{F}_{q}$. In particular, if $g \in G_{\Phi_{6}(q)}$, then

$$
g^{q^{3}+1}=g^{q^{2}-q+1}=1
$$

and using (3.3) together with (3.1) and (3.2) we obtain the following nine relations for $g_{i}$ 's:

$$
\begin{array}{ll}
P_{1}: & 2 g_{0} g_{4}-g_{2}^{2}-\left(2 g_{1} g_{5}-g_{3}^{2}\right) c=0, \\
P_{2}: & -2 g_{1} g_{2}+g_{4}^{2}+2 g_{0} g_{3}-g_{5}^{2} c=0, \\
P_{3}: & g_{0}^{2}-1-\left(g_{1}^{2}+2 g_{2} g_{5}-2 g_{3} g_{4}\right) c=0, \\
P_{4}: & \left(1-g_{0}\right) g_{5}+2 g_{2} g_{3}-g_{1} g_{4}=0, \\
P_{5}: & \left(-1-g_{0}\right) g_{4}+g_{2}^{2}+\left(g_{3}^{2}-g_{1} g_{5}\right) c=0, \\
P_{6}: & \left(g_{0}+1\right) g_{3}+g_{1} g_{2}-g_{4}^{2}-g_{5}^{2} c=0, \\
P_{7}: & \left(g_{0}-1\right) g_{2}+\left(g_{3} g_{1}-2 g_{5} g_{4}\right) c=0, \\
P_{8}: & \left(1+2 g_{0}\right) g_{1}-g_{2} g_{4}-g_{3} g_{5} c=0, \\
P_{9}: & g_{0}\left(g_{0}-1\right)+\left(g_{1}^{2}-g_{2} g_{5}-g_{3} g_{4}\right) c=0 .
\end{array}
$$

Note that each relation $P_{i}$ is independent of the choice of the primitive sixth root of unity $m$. Therefore, without loss of generality, we associate a 7-variate polynomial $P_{i}(X)=P_{i}\left(x_{0}, \ldots, x_{5}, y\right)$ to each relation $P_{i}$ above. That is, if $g=$ $\left(g_{0}+g_{1} w\right)+\left(g_{2}+g_{3} w\right) \sigma+\left(g_{4}+g_{5} w\right) \sigma^{2} \in G_{\Phi_{6}(q)}$, then $P_{i}\left(g_{0}, \ldots, g_{5}, c\right)=0$ for all $i=1, \ldots, 9$. Thus, every element in $G_{\Phi_{6}(q)}$ corresponds to an $\mathbb{F}_{q}$-point in the variety defined by the ideal $\left\langle P_{1}(X), \ldots, P_{9}(X)\right\rangle$.

In fact, we may think of each $P_{i}(X)$ as a 7 -variate polynomial defined over the field of rational numbers $\mathbb{Q}$, and define an ideal $\mathcal{I}=\left\langle P_{1}(X), \ldots, P_{9}(X)\right\rangle$ over $\mathbb{Q}$. 
Next, we compute a Groebner basis over $\mathbb{Q}$ of $\mathcal{I}$ with respect to some fixed ordering on the set of monomials, which in turn yields a factor- $3 / 2$ compression function $\mathcal{C}$ for elements $g \in G_{\Phi_{6}(q)}$ with the property that given $\mathcal{C}(g), g$ can be recovered uniquely at a cost dominated by an inversion in $\mathbb{F}_{q}$. We describe the compression and decompression functions in the following theorem.

Theorem 3.1. Let $q \equiv 1(\bmod 6)$ be a prime power. Let $\mathbb{F}_{q^{2}}=\mathbb{F}_{q}(w)$ where $w^{2}=c, c \in \mathbb{F}_{q}$ is a quadratic non-residue, $w \in \mathbb{F}_{q^{2}}$ is a cubic non-residue; and let $\mathbb{F}_{q^{6}}=\mathbb{F}_{q^{2}}(\sigma)$ where $\sigma^{3}=w$. Let $g=\left(g_{0}+g_{1} w\right)+\left(g_{2}+g_{3} w\right) \sigma+\left(g_{4}+g_{5} w\right) \sigma^{2} \in$ $G_{\Phi_{6}(q)} \backslash\{1\} \subset \mathbb{F}_{q^{6}}^{*}$. Define the compression function $\mathcal{C}$ and the decompression function $\mathcal{D}$ as follows:

$$
\begin{aligned}
\mathcal{C}(g) & =\left[g_{2}, g_{3}, g_{4}, g_{5}\right] \\
\mathcal{D}\left(\left[\tilde{g}_{2}, \tilde{g}_{3}, \tilde{g}_{4}, \tilde{g}_{5}\right]\right) & =\left(\tilde{g}_{0}+\tilde{g}_{1} w\right)+\left(\tilde{g}_{2}+\tilde{g}_{3} w\right) \sigma+\left(\tilde{g}_{4}+\tilde{g}_{5} w\right) \sigma^{2},
\end{aligned}
$$

where

$$
\begin{cases}\tilde{g}_{1}=\frac{\tilde{g}_{5}^{2} c+3 \tilde{g}_{4}^{2}-2 \tilde{g}_{3}}{4 \tilde{g}_{2}}, \quad \tilde{g}_{0}=\left(2 \tilde{g}_{1}^{2}+\tilde{g}_{2} \tilde{g}_{5}-3 \tilde{g}_{3} \tilde{g}_{4}\right) c+1, & \text { if } \tilde{g}_{2} \neq 0 ; \\ \tilde{g}_{1}=\frac{2 \tilde{g}_{4} \tilde{g}_{5}}{\tilde{g}_{3}}, \quad \tilde{g}_{0}=\left(2 \tilde{g}_{1}^{2}-3 \tilde{g}_{3} \tilde{g}_{4}\right) c+1, & \text { if } \tilde{g}_{2}=0 .\end{cases}
$$

Then $\mathcal{D}$ is well-defined for all $\mathcal{C}(g)$ with $g \in G_{\Phi_{6}(q)} \backslash\{1\}$, and $\mathcal{D}(\mathcal{C}(g))=$ g for all $g \in G_{\Phi_{6}(q)} \backslash\{1\}$.

Proof. Let $g=\left(g_{0}+g_{1} w\right)+\left(g_{2}+g_{3} w\right) \sigma+\left(g_{4}+g_{5} w\right) \sigma^{2} \in G_{\Phi_{6}(q)} \backslash\{1\}$. If $g_{2}=0$ and $g_{3}=0$ then one can verify using the relations $P_{i}$ that $g_{1}=g_{4}=g_{5}=0$ and $g_{0}=1$. Therefore, $g_{2}$ and $g_{3}$ cannot both be zero proving that $\mathcal{D}$ is well-defined for all $\mathcal{C}(g)$ with $g \in G_{\Phi_{6}(q)} \backslash\{1\}$.

To show that $\mathcal{D}(\mathcal{C}(g))=g$ for all $g \in G_{\Phi_{6}(q)} \backslash\{1\}$, we compute a Groebner basis over $\mathbb{Q}$ of the ideal $\mathcal{I}=\left\langle P_{1}(X), \ldots P_{9}(X)\right\rangle$ with respect to the lexicographical ordering of the monomials with $x_{0}>x_{1}>x_{5}>x_{4}>x_{3}>x_{2}>y$. It can be verified using Magma with the commands

$$
\begin{aligned}
& \left.\mathrm{R}<x_{0}, x_{1}, x_{5}, x_{4}, x_{3}, x_{2}, y>:=\text { PolynomialRing(RationalField }(), 7\right) \\
& \mathrm{B} 1:=\left[\mathrm{R} ! P_{1}, \mathrm{R} ! P_{2}, \mathrm{R} ! P_{3}, \mathrm{R} ! P_{4}, \mathrm{R} ! P_{5}, \mathrm{R} ! P_{6}, \mathrm{R} ! P_{7}, \mathrm{R} ! P_{8}, \mathrm{R} ! P_{9}\right] \\
& \mathrm{I} 1:=\text { ideal }<\mathrm{R} \mid \mathrm{B} 1>; \\
& \mathrm{GB} 1:=\text { GroebnerBasis(I1); } \\
& \mathrm{B} 2:=\left[\mathrm{R} ! P_{1}, \mathrm{R} ! P_{2}, \mathrm{R} ! P_{3}, \mathrm{R} ! P_{4}, \mathrm{R} ! P_{5}, \mathrm{R} ! P_{6}, \mathrm{R} ! P_{7}, \mathrm{R} ! P_{8}, \mathrm{R} ! P_{9}, \mathrm{R} ! x_{2}\right] \\
& \mathrm{I} 2:=\text { ideal }<\mathrm{R} \mid \mathrm{B} 2>; \\
& \mathrm{GB} 2:=\text { GroebnerBasis }(\mathrm{I} 2)
\end{aligned}
$$

that

$$
\begin{array}{r}
x_{0}-\left(2 x_{1}^{2}+x_{2} x_{5}-3 x_{3} x_{4}\right) y-1, \\
x_{1} x_{2}+\frac{x_{3}}{2}-\frac{3 x_{4}^{2}}{4}-\frac{x_{5}^{2} y}{4}
\end{array}
$$

are two polynomials in the basis GB1; and

$$
x_{1} x_{3}-2 x_{4} x_{5}
$$

is a polynomial in the basis GB2. 
By Theorem 3.1, we know that if $g=\left(g_{0}+g_{1} w\right)+\left(g_{2}+g_{3} w\right) \sigma+\left(g_{4}+g_{5} w\right) \sigma^{2} \in$ $G_{\Phi_{6}(q)}$ then $\mathcal{C}(g)=\left[g_{2}, g_{3}, g_{4}, g_{5}\right]$ determines $g$ uniquely. This suggests a squaring formula that uses the compressed representation of elements in $G_{\Phi_{6}(q)}$ which we present in Theorem 3.2 .

Theorem 3.2. Let $q \equiv 1(\bmod 6)$ be a prime power. Let $\mathbb{F}_{q^{2}}=\mathbb{F}_{q}(w)$ where $w^{2}=$ $c, c \in \mathbb{F}_{q}$ is a quadratic non-residue, $w \in \mathbb{F}_{q^{2}}$ is a cubic non-residue; and let $\mathbb{F}_{q^{6}}=$ $\mathbb{F}_{q^{2}}(\sigma)$ where $\sigma^{3}=w$. Let $g=\left(g_{0}+g_{1} w\right)+\left(g_{2}+g_{3} w\right) \sigma+\left(g_{4}+g_{5} w\right) \sigma^{2} \in G_{\Phi_{6}(q)} \subset \mathbb{F}_{q^{6}}^{*}$. Let $\mathcal{C}$ be the compression function defined in the statement of Theorem 3.1. Let $h=g^{2}$, where $h=\left(h_{0}+h_{1} w\right)+\left(h_{2}+h_{3} w\right) \sigma+\left(h_{4}+h_{5} w\right) \sigma^{2}$. Then

$$
\mathcal{C}\left(g^{2}\right)=\left[h_{2}, h_{3}, h_{4}, h_{5}\right]
$$

where

$$
\begin{aligned}
h_{2} & =2\left(g_{2}+3 c B_{4,5}\right), \\
h_{3} & =3\left(A_{4,5}-(c+1) B_{4,5}\right)-2 g_{3}, \\
h_{4} & =3\left(A_{2,3}-(c+1) B_{2,3}\right)-2 g_{4}, \\
h_{5} & =2\left(g_{5}+3 B_{2,3}\right), \\
A_{i, j} & =\left(g_{i}+g_{j}\right)\left(g_{i}+c g_{j}\right), \\
B_{i, j} & =g_{i} g_{j} .
\end{aligned}
$$

Proof. Using $w^{2}=c$ and $\sigma^{3}=w$, we find that

$$
\begin{aligned}
h_{0} & =g_{0}^{2}+\left(g_{1}^{2}+2 g_{3} g_{4}+2 g_{2} g_{5}\right) c \\
h_{1} & =2\left(g_{0} g_{1}+g_{2} g_{4}+g_{3} g_{5} c\right), \\
h_{2} & =2\left(g_{0} g_{2}+\left(g_{4} g_{5}+g_{1} g_{3}\right) c\right), \\
h_{3} & =2\left(g_{0} g_{3}+g_{1} g_{2}\right)+g_{4}^{2}+g_{5}^{2} c, \\
h_{4} & =g_{2}^{2}+2 g_{0} g_{4}+\left(g_{3}^{2}+2 g_{1} g_{5}\right) c \\
h_{5} & =2\left(g_{0} g_{5}+g_{2} g_{3}+g_{1} g_{4}\right) .
\end{aligned}
$$

Under the correspondence $x_{i} \leftrightarrow g_{i}$ and $y \leftrightarrow c$, we define the 7-variate polynomials $h_{i}(X)$. Next, we compute representatives of $h_{i}(X)$ 's for $i \in\{2,3,4,5\}$ in the quotient ring $R / \mathcal{I}$, where $R=\mathbb{Q}\left[x_{0}, \ldots, x_{5}, y\right]$ with respect to the lexicographical ordering of monomials with $x_{0}>x_{1}>x_{5}>x_{4}>x_{3}>x_{2}>y$, and where $\mathcal{I}=\left\langle P_{1}(X), \ldots, P_{9}(X)\right\rangle$ is the ideal over $\mathbb{Q}$ defined earlier. It can be verified using Magma with the commands:

$$
\begin{aligned}
& \left.\mathrm{R}<x_{0}, x_{1}, x_{5}, x_{4}, x_{3}, x_{2}, y>:=\text { PolynomialRing(RationalField }(), 7\right) \\
& \mathrm{B}:=\left[\mathrm{R} ! P_{1}, \mathrm{R} ! P_{2}, \mathrm{R} ! P_{3}, \mathrm{R} ! P_{4}, \mathrm{R} ! P_{5}, \mathrm{R} ! P_{6}, \mathrm{R} ! P_{7}, \mathrm{R} ! P_{8}, \mathrm{R} ! P_{9}\right] \\
& \mathrm{I}:=\text { ideal }<\mathrm{R} \mid \mathrm{B}> \\
& \mathrm{R} 2:=\mathrm{R} / \mathrm{I} ; \\
& \mathrm{R} 2 ! \mathrm{h}_{2} ; \mathrm{R} 2 ! \mathrm{h}_{3} ; \mathrm{R} 2 ! \mathrm{h}_{4} ; \mathrm{R} 2 ! \mathrm{h}_{5} ;
\end{aligned}
$$

that

$$
\begin{aligned}
& h_{2}=2\left(g_{2}+3 g_{4} g_{5} c\right)=2\left(g_{2}+3 c B_{4,5}\right), \\
& h_{3}=3\left(g_{4}^{2}+g_{5}^{2} c\right)-2 g_{3}=3\left(A_{4,5}-(c+1) B_{4,5}\right)-2 g_{3}, \\
& h_{4}=3\left(g_{2}^{2}+g_{3}^{2} c\right)-2 g_{4}=3\left(A_{2,3}-(c+1) B_{2,3}\right)-2 g_{4}, \\
& h_{5}=2\left(g_{5}+3 g_{2} g_{3}\right)=2\left(g_{5}+3 B_{2,3}\right)
\end{aligned}
$$

in the quotient ring $R / \mathcal{I}$. 
Corollary 3.3. Let $q \equiv 1(\bmod 6)$ and $g \in G_{\Phi_{6}(q)} \subset \mathbb{F}_{q^{6}}^{*}$. Then $\mathcal{C}\left(g^{2}\right)$ can be computed at a cost dominated by 4 multiplications in $\mathbb{F}_{q}$.

\section{Applications}

The squaring formula in Theorem 3.2 works with a compressed representation of elements in $G_{\Phi_{6}(q)}$. The decompression cost for this representation is far less than that of other compressed-squaring algorithms such as XTR-squaring (LUCsquaring) where decompression is performed by finding a root of a third (second) degree irreducible polynomial defined over $\mathbb{F}_{q^{2}}\left(\mathbb{F}_{q^{3}}\right)$. When the full representation of a compressed element $\mathcal{C}(g)$ is needed (for example, in the multiplication step of square-and-multiply type exponentiation algorithms), the decompression function $\mathcal{D}$ can recover $g$ uniquely at a cost dominated by an inversion in $\mathbb{F}_{q}$. Consequently, comparing Corollary 3.3 with Table 1, our compressed-squaring algorithm together with the decompression function yields an exponentiation algorithm which is especially fast when the exponent has low Hamming weight.

4.1. Exponentiation in $G_{\Phi_{6}(q)}$. Let $q=p^{i} \equiv 1(\bmod 6)$ and $g \in G_{\Phi_{6}(q)} \subset$ $\mathbb{F}_{q^{6}}^{*}$. We present an exponentiation algorithm that uses the squaring formula in Theorem 3.2

Let $e$ be an $\ell$-bit exponent with binary representation

$$
e=e_{\ell-1} e_{\ell-2} \ldots e_{2} e_{1} e_{0}
$$

where $e_{\ell-1}=1$. Let $H_{e}=\left\{i: 1 \leq i \leq \ell-1\right.$ and $\left.e_{i}=1\right\}$. Then

$$
g^{e}=\prod_{i=0}^{\ell-1} g^{2^{i}}=g^{e_{0}} \prod_{i \in H_{e}} \mathcal{D}\left(\mathcal{C}\left(g^{2^{i}}\right)\right) .
$$

Now, if

$$
g^{2^{i}}=\left(g_{i, 0}+g_{i, 1} w\right)+\left(g_{i, 2}+g_{i, 3} w\right) \sigma+\left(g_{i, 4}+g_{i, 5} w\right) \sigma^{2}
$$

then by Theorem 3.1 ,

$$
\mathcal{C}\left(g^{2^{i}}\right)=\left[g_{i, 2}, g_{i, 3}, g_{i, 4}, g_{i, 5}\right]
$$

and, assuming without loss of generality that $g_{i, 2} \neq 0$,

$$
\mathcal{D}\left(\mathcal{C}\left(g^{2^{i}}\right)\right)=\left(x_{i}+\frac{y_{i}}{z_{i}} w\right)+\left(g_{i, 2}+g_{i, 3} w\right) \sigma+\left(g_{i, 4}+g_{i, 5} w\right) \sigma^{2},
$$

where

$$
x_{i}=\left(2 g_{i, 1}^{2}+g_{i, 2} g_{i, 5}-3 g_{i, 3} g_{i, 4}\right) c+1, y_{i}=g_{i, 5}^{2} c+3 g_{i, 4}^{2}-2 g_{i, 3} \text {, and } z_{i}=4 g_{i, 2} \text {. }
$$

Hence, $g^{e}$ can be computed as follows:

(1) Compute $\mathcal{C}\left(g^{2^{i}}\right)$ for $1 \leq i \leq \ell-1$ with $(\ell-1)$ successive squarings using Theorem 3.2 and store $\mathcal{C}\left(g^{2^{i}}\right)$ for each $i \in H_{e}$.

(2) Compute and store $\left(x_{i}, y_{i}, z_{i}\right)$ for each $i \in H_{e}$.

(3) Compute $y_{i} / z_{i}$ for each $i \in H_{e}$.

(4) Compute $g^{e}=g^{e_{0}} \prod_{i \in H_{e}} \mathcal{D}\left(\mathcal{C}\left(g^{2^{i}}\right)\right)$.

Now, let $\left|H_{e}\right|=N$, and let $M_{i}, S_{i}$ and $I_{i}$ denote multiplication, squaring and inversion costs in $\mathbb{F}_{q}=\mathbb{F}_{p^{i}}$, respectively. By Corollary 3.3] step (10) in the above algorithm has cost dominated by $(4(\ell-1)) M_{i}$ and requires storage of $4 N \mathbb{F}_{q^{-}}$ elements. Using Montgomery's simultaneous inversion trick [20, 13, steps (2) and (3) have cost dominated by $N\left(\left(1 S_{i}+2 M_{i}\right)+2 S_{i}\right)+3(N-1) M_{i}+1 I_{i}+(N) M_{i}$ 
and storage of $3 N+1 \mathbb{F}_{q}$-elements. Finally, step (4) can be computed at a cost of $(N) M_{6 i}$.

Corollary 4.1. Let $q=p^{i} \equiv 1(\bmod 6)$ and $g \in G_{\Phi_{6}(q)} \subset \mathbb{F}_{q^{6}}$. Let e be an $\ell$-bit exponent. Let $H_{e}=\left\{i: 1 \leq i \leq \ell-1\right.$ and $\left.e_{i}=1\right\}$, and let $\left|H_{e}\right|=N$. Then, $g^{e}$ can be computed at a cost dominated by

$$
(4(\ell-1)) M_{i}+(6 N-3) M_{i}+(N) M_{6 i}+(3 N) S_{i}+1 I_{i},
$$

with a storage of $7 N+1 \mathbb{F}_{q}$-elements.

Note that the cost of exponentiation using GS-squaring (see [12] or Section 22) in the same setting as in Corollary 4.1 would be dominated by

$$
6(\ell-1) M_{i}+(N) M_{6 i} .
$$

Hence, by Corollary 4.1, we would expect a 33\% speed-up over GS-exponentiation as $N / \ell \rightarrow 0$.

4.2. Speeding up pairing computations. Let $E: y^{2}=x^{3}+b$ be a curve in the Barreto-Naehrig (BN) family of pairing-friendly curves with embedding degree $k=12\left[2\right.$. Then $E$ is defined over $\mathbb{F}_{p}$ with $\left|E\left(\mathbb{F}_{p}\right)\right|=r$, where the primes $p$ and $r$ are parametrized as follows:

$$
\begin{aligned}
& p(u)=36 u^{4}+36 u^{3}+24 u^{2}+6 u+1, \\
& r(u)=36 u^{4}+36 u^{3}+18 u^{2}+6 u+1 .
\end{aligned}
$$

In general, a pairing computation on $E$ is performed in two steps: Miller loop and final exponentiation. Scott 24] showed that the final exponentiation in the pairing computation can be done in two parts. In the first part, an element in $\mathbb{F}_{p^{12}}^{*}$ is raised to the power $\left(p^{6}-1\right)\left(p^{2}+1\right)$. This is the so-called easy part and requires a small number of multiplications, $p$ th powerings and a single inversion, and yields an element $g \in G_{\Phi_{6}\left(p^{2}\right)}=G_{\Phi_{12}(p)} \subset \mathbb{F}_{p^{12}}^{*}$. In the second part of the final exponentiation, the so-called hard part, $g$ is raised to the power

$$
\Phi_{12}(p) / r=\left(p^{4}-p^{2}+1\right) / r=\lambda_{3} p^{3}+\lambda_{2} p^{2}+\lambda_{1} p+\lambda_{0},
$$

where

$$
\begin{aligned}
& \lambda_{3}(u)=1, \\
& \lambda_{2}(u)=6 u^{2}+1, \\
& \lambda_{1}(u)=-36 u^{3}-18 u^{2}-12 u+1, \\
& \lambda_{0}(u)=-36 u^{3}-30 u^{2}-18 u-2 .
\end{aligned}
$$

Note that when the BN parameter $u$ is chosen to have low Hamming weight, one can first compute $g^{u}, g^{u^{2}}=\left(g^{u}\right)^{u}$ and $g^{u^{3}}=\left(g^{u^{2}}\right)^{u}$ to minimize the number of multiplications in the hard part of the final exponentiation.

To be more concrete, one can choose $u=-\left(2^{62}+2^{55}+1\right)$ to obtain a BNcurve $E$ defined over a 254-bit prime $p$ with 254-bit prime order group $E\left(\mathbb{F}_{p}\right)$ 21. In our operation counts, we will assume: a) $M_{2}=3 M_{1}$ and $M_{12}=54 M_{1}$ using Karatsuba's technique; b) $S_{2}=2 M_{1}$ (see general squaring in Section 2.2); c) $I_{2}=1 I_{1}+2 M_{1}+2 S_{1}[19]$; and $\left.d\right) M_{1}=S_{1}$ and $I_{1}=50 M_{1}$ (see [4, Section 3.1]).

GS-exponentiation seems to be the fastest of the previously-known methods to compute the hard part of the final exponentiation in the pairing computation. Using 
this method, each of $g^{-u}, g^{-u^{2}}$ and $g^{-u^{3}}$ can be computed at a cost dominated by $($ see (4.1))

$$
6(\ell-1) M_{i}+(N) M_{6 i}=1224 M_{1},
$$

where $\ell=63$ and $N=2$.

Now, we describe an exponentiation algorithm similar to the one in Section 4.1 but avoiding the storage requirements. Instead of performing simultaneous inversion, we begin the algorithm by computing compressed-squarings and perform a one-time inversion (decompression) when a multiplication is required. Then we switch to GS-squaring. In summary, given $g$ and $-u=2^{62}+2^{55}+1, g^{-u}$ can be computed as follows:

(1) Compute $\mathcal{C}\left(g^{2^{55}}\right)$ with 55 successive squarings using Theorem 3.2

(2) Decompress $\mathcal{C}\left(g^{2^{55}}\right)$ to obtain $g^{2^{55}}=\mathcal{D}\left(\mathcal{C}\left(g^{2^{55}}\right)\right)$.

(3) Compute $g^{2^{62}}$ with 7 successive GS-squarings.

(4) Compute $g^{-u}=g \cdot g^{2^{55}} \cdot g^{2^{62}}$.

The cost of step (2) is dominated by

$$
\left(3 S_{2}+2 M_{2}\right)+1 I_{2}+1 M_{2}=6 M_{1}+6 M_{1}+54 M_{1}+3 M_{1}=69 M_{1} .
$$

Similar to our previous analysis, one can verify that the cost of the above hybrid exponentiation algorithm is dominated by

$$
(55 \cdot 4) M_{2}+(7 \cdot 6) M_{2}+69 M_{1}+2 M_{12}=963 M_{1} \text {. }
$$

Comparing (4.2) and (4.3), we would expect around a $21 \%$ speed-up for computing $g^{u}, g^{u^{2}}$ and $g^{u^{3}}$ in the hard part of the final exponentiation. According to 4, Section 4.2 and Table 3], computing $g^{u}, g^{u^{2}}$ and $g^{u^{3}}$ take $79 \%$ of the time of the final exponentiation, and the final exponentiation takes $42 \%$ of the time of the whole pairing computation. Hence, with our new exponentiation algorithm, we would expect a $17 \%$ speed-up for the final exponentiation, and a $7 \%$ speed-up for the pairing computation.

Recently, Aranha et al. proposed and implemented a variant of the squaring algorithm in Theorem 3.2 for the pairing computation over a BN curve parametrized by $u=-\left(2^{62}+2^{55}+1\right)[1$. They reported overall $5 \%-7 \%$ speed-ups (gained from using this new squaring algorithm) for the pairing computation.

\section{OTHER FORMULAE FOR SQUARING}

In this section, we describe a general method for finding efficient squaring formulae in cyclotomic subgroups $G_{\Phi_{6}(q)}$. While rediscovering some of the previouslyknown squaring formulae such as LUC-squaring, XTR-squaring and GS-squaring, our method yields new squaring formulae which might be good alternatives to the one in Section 3.

We use the same notation as in Section 3, In particular, let

$$
g=\left(g_{0}+g_{1} w\right)+\left(g_{2}+g_{3} w\right) \sigma+\left(g_{4}+g_{5} w\right) \sigma^{2} \in G_{\Phi_{6}(q)}
$$

and

$$
h=g^{2}=\left(h_{0}+h_{1} w\right)+\left(h_{2}+h_{3} w\right) \sigma+\left(h_{4}+h_{5} w\right) \sigma^{2} .
$$

Let $I \subseteq\{0,1, \ldots, 5\}$. In order to obtain squaring formulae similar to the one in Theorem 3.2 we first need a (compression) function $\mathcal{C}$ such that $\mathcal{C}(g)$ can be determined as a function of $V_{I}(g)=\left\{g_{i}: i \in I\right\}$. Second, we need a family 
$\mathcal{S}_{I}=\left\{S_{i}: i \in I\right\}$ of formulae to compute each $h_{i} \in V_{I}(h)$ as a function of $V_{I}(g)$, say $h_{i}=S_{i}\left(V_{I}(g)\right)$ for each $i \in I$. Consequently, we might represent a squaring formula $\mathcal{F}: \mathcal{C}(g) \mapsto \mathcal{C}\left(g^{2}\right)=\mathcal{C}(h)$ by a tuple

$$
\mathcal{F}=\left\{I, \mathcal{C}, \mathcal{S}_{I}\right\}
$$

For example, in Theorem 3.2 we had

$$
\begin{aligned}
I & =\{2,3,4,5\}, \mathcal{C}(g)=\left[g_{2}, g_{3}, g_{4}, g_{5}\right], \mathcal{S}_{I}=\left\{S_{2}, S_{3}, S_{4}, S_{5}\right\}, \\
h_{2} & =S_{2}\left(V_{I}(g)\right)=2\left(g_{2}+3 g_{4} g_{5} c\right), \\
h_{3} & =S_{3}\left(V_{I}(g)\right)=3\left(g_{4}^{2}+g_{5}^{2} c\right)-2 g_{3}, \\
h_{4} & =S_{4}\left(V_{I}(g)\right)=3\left(g_{2}^{2}+g_{3}^{2} c\right)-2 g_{4}, \\
h_{5} & =S_{5}\left(V_{I}(g)\right)=2\left(g_{5}+3 g_{2} g_{3}\right) .
\end{aligned}
$$

Remark 5.1. In the representation of $\mathcal{F}$, it seems necessary to require that the inverse of $\mathcal{C}$ can be efficiently computed in order to get efficient multi-exponentiation algorithms based on $\mathcal{F}$. However, we will relax this condition for now.

We already know from the proof of Theorem 3.2 that if $h=g^{2}$, then

$$
\begin{aligned}
h_{0} & =g_{0}^{2}+\left(g_{1}^{2}+2 g_{3} g_{4}+2 g_{2} g_{5}\right) c, \\
h_{1} & =2\left(g_{0} g_{1}+g_{2} g_{4}+g_{3} g_{5} c\right), \\
h_{2} & =2\left(g_{0} g_{2}+\left(g_{4} g_{5}+g_{1} g_{3}\right) c\right), \\
h_{3} & =2\left(g_{0} g_{3}+g_{1} g_{2}\right)+g_{4}^{2}+g_{5}^{2} c, \\
h_{4} & =g_{2}^{2}+2 g_{0} g_{4}+\left(g_{3}^{2}+2 g_{1} g_{5}\right) c, \\
h_{5} & =2\left(g_{0} g_{5}+g_{2} g_{3}+g_{1} g_{4}\right) .
\end{aligned}
$$

In fact, the squaring formula in Theorem 3.2 , or, in other words,

$$
\mathcal{S}_{I}=\left\{S_{2}, S_{3}, S_{4}, S_{5}\right\},
$$

was found by computing representatives of $h_{i}(X)$ 's for $i \in\{2,3,4,5\}$ in the quotient ring $R / \mathcal{I}$, where $R=\mathbb{Q}\left[x_{0}, \ldots, x_{5}, y\right]$ with respect to the lexicographical ordering of monomials with $x_{0}>x_{1}>x_{5}>x_{4}>x_{3}>x_{2}>y$, and where $\mathcal{I}=\left\langle P_{1}(X), \ldots, P_{9}(X)\right\rangle$.

In order to capture a wider class of squaring formulae $\mathcal{F}=\left\{I, \mathcal{C}, \mathcal{S}_{I}\right\}$, we will compute representatives of $h_{i}(X)$ for $i \in\{0, \ldots, 5\}$ in the quotient ring $R / \mathcal{I}$ by varying over all the $7 !=5040$ orderings of the variables $\left\{x_{0}, \ldots, x_{5}, y\right\}$. To do so, we let $o$ be some fixed ordering in the set $\mathcal{O}$ of all orderings of the variables $\left\{x_{0}, \ldots, x_{5}, y\right\}$ and denote by $R_{o}$ the ring $\mathbb{Q}\left[x_{0}, \ldots, x_{5}, y\right]$ with respect to the lexicographical ordering of monomials with ordering $o$.

We define

$$
\mathcal{H}_{i}=\left\{\bar{h}_{i, o}: \bar{h}_{i, o}=h_{i} \in R_{o} / \mathcal{I}, o \in \mathcal{O}\right\} .
$$

Since each $\bar{h}_{i, o}$ defines a unique $S_{i}$ (on some subset $V_{I}(g)$ ), we may replace, without loss of generality, $\bar{h}_{i, o}$ 's by $S_{i, j}$ 's in $\mathcal{H}_{i}$. We list $\mathcal{H}_{i}$ for $i=0,1, \ldots, 5$ in Appendix A

Note that for any $I \subseteq\{0,1,2 \ldots, 5\}$, there is a squaring formula $\mathcal{F}=\left\{I, \mathcal{C}, \mathcal{S}_{I}\right\}$ only if for each $i \in I$ there is some $S_{i, j} \in \mathcal{H}_{i}$ that is defined on $V_{I}(g)$. From Appendix A, we deduce that $S_{0, j} \in \mathcal{H}_{0}$ is well defined on a domain $V_{I}(g)$ only if $V_{I}(g)$ contains one of the subsets in the minimal domain set $D_{0}$ of $\mathcal{H}_{0}$, where 


$$
\begin{aligned}
D_{0}= & \left\{\left\{g_{0}, g_{1}\right\},\left\{g_{0}, g_{2}, g_{5}\right\},\left\{g_{0}, g_{3}, g_{4}\right\},\left\{g_{1}, g_{2}, g_{5}\right\},\left\{g_{0}, g_{1}, g_{2}, g_{3}\right\},\right. \\
& \left.\left\{g_{0}, g_{1}, g_{3}, g_{4}\right\},\left\{g_{0}, g_{1}, g_{4}, g_{5}\right\},\left\{g_{0}, g_{2}, g_{3}, g_{4}, g_{5}\right\}\right\} .
\end{aligned}
$$

Similarly, we have

$$
\begin{aligned}
D_{1}= & \left\{\left\{g_{0}, g_{1}\right\},\left\{g_{1}, g_{2}, g_{5}\right\},\left\{g_{1}, g_{2}, g_{3}, g_{4}\right\},\left\{g_{1}, g_{2}, g_{3}, g_{5}\right\},\right. \\
& \left\{g_{1}, g_{2}, g_{4}, g_{5}\right\},\left\{g_{1}, g_{3}, g_{4}, g_{5}\right\},\left\{g_{0}, g_{1}, g_{2}, g_{3}, g_{5}\right\}, \\
& \left.\left\{g_{0}, g_{1}, g_{2}, g_{4}, g_{5}\right\},\left\{g_{1}, g_{2}, g_{3}, g_{4}, g_{5}\right\}\right\}, \\
D_{2}= & \left\{\left\{g_{2}, g_{4}, g_{5}\right\},\left\{g_{0}, g_{1}, g_{2}, g_{3}\right\},\left\{g_{1}, g_{2}, g_{3}, g_{5}\right\},\left\{g_{1}, g_{2}, g_{3}, g_{4}, g_{5}\right\}\right\}, \\
D_{3}= & \left\{\left\{g_{0}, g_{3}, g_{4}\right\},\left\{g_{0}, g_{3}, g_{5}\right\},\left\{g_{1}, g_{2}, g_{5}\right\},\left\{g_{3}, g_{4}, g_{5}\right\},\right. \\
& \left.\left\{g_{0}, g_{1}, g_{2}, g_{3}\right\},\left\{g_{1}, g_{2}, g_{3}, g_{4}\right\},\left\{g_{1}, g_{2}, g_{3}, g_{5}\right\}\right\}, \\
D_{4}= & \left\{\left\{g_{0}, g_{2}, g_{4}\right\},\left\{g_{0}, g_{3}, g_{4}\right\},\left\{g_{1}, g_{2}, g_{5}\right\},\left\{g_{2}, g_{3}, g_{4}\right\},\right. \\
& \left.\left\{g_{0}, g_{1}, g_{4}, g_{5}\right\},\left\{g_{1}, g_{2}, g_{4}, g_{5}\right\},\left\{g_{1}, g_{3}, g_{4}, g_{5}\right\}\right\}, \\
D_{5}= & \left\{\left\{g_{2}, g_{3}, g_{5}\right\},\left\{g_{0}, g_{1}, g_{4}, g_{5}\right\},\left\{g_{1}, g_{2}, g_{4}, g_{5}\right\},\left\{g_{1}, g_{2}, g_{3}, g_{4}, g_{5}\right\}\right\} .
\end{aligned}
$$

Now, from the minimal domain sets $D_{i}$ 's and $\mathcal{H}_{i}$ 's in Appendix $\mathrm{A}$ we can write other squaring formulae $\mathcal{F}=\left\{I, \mathcal{C}, \mathcal{S}_{I}\right\}$ as follows.

5.1. $\mathbf{S Q R}_{01}: \mathcal{F}=\left\{I, \mathcal{C}, \mathcal{S}_{I}\right\}$ with $|I|=2$.

$$
\begin{aligned}
I & =\{0,1\}, \mathcal{C}(g)=\left[g_{0}, g_{1}\right], \mathcal{S}_{I}=\left\{S_{0,3}, S_{1,1}\right\}, \\
h_{0} & =S_{0,3}=3 g_{0}^{2}-2 g_{0}+3 g_{1}^{2} c, \\
h_{1} & =S_{1,1}=6 g_{0} g_{1}+2 g_{1} .
\end{aligned}
$$

This formula is the only one with $|I|=2$. Its cost is dominated by 2 multiplications in $\mathbb{F}_{q}$ as one can write

$$
h_{0}=3\left(\left(g_{0}+g_{1}\right)\left(g_{0}+g_{1} c\right)-(c+1) g_{0} g_{1}\right)-2 g_{0} .
$$

In fact, this formula is a rediscovery of the XTR-squaring because

$$
\operatorname{Tr}_{\mathbb{F}_{q^{6}} / \mathbb{F}_{q^{2}}}(g)=3\left(g_{0}+g_{1} w\right)
$$

can be uniquely determined using $V_{I}(g)=\left\{g_{0}, g_{1}\right\}$. Note that the compression function $\mathcal{C}$ in this case cannot have an inverse as $\mathcal{C}(g)=\mathcal{C}\left(g^{q^{2}}\right)=\mathcal{C}\left(g^{q^{4}}\right)$.

5.2. $\mathbf{S Q R}_{034}: \mathcal{F}=\left\{I, \mathcal{C}, \mathcal{S}_{I}\right\}$ with $|I|=3$.

$$
\begin{aligned}
I & =\{0,3,4\}, \mathcal{C}(g)=\left[g_{0}, g_{3}, g_{4}\right], \mathcal{S}_{I}=\left\{S_{0,1}, S_{3,2}, S_{4,1}\right\}, \\
h_{0} & =S_{0,1}=2 g_{0}^{2}+4 g_{3} g_{4} c-1, \\
h_{3} & =S_{3,2}=4 g_{0} g_{3}+2 g_{4}^{2} \\
h_{4} & =S_{4,1}=4 g_{0} g_{4}+2 g_{3}^{2} c .
\end{aligned}
$$

This formula is the only one with $|I|=3$, and is a rediscovery of the LUC-squaring because

$$
\operatorname{Tr}_{\mathbb{F}_{q^{6}} / \mathbb{F}_{q^{3}}}(g)=2\left(g_{0}+g_{3} w \sigma+g_{4} \sigma^{2}\right)
$$

can be uniquely determined using $V_{I}(g)=\left\{g_{0}, g_{3}, g_{4}\right\}$. In fact, using

$$
\operatorname{Tr}_{q^{6}, q^{3}}\left(g^{2}\right)=\operatorname{Tr}_{q^{6}, q^{3}}(g)^{2}-2
$$


and the 3 -way squaring formula in [6] one can show that

$$
\begin{aligned}
& h_{0}=2\left(T_{0}+T_{1} c\right)-1, \\
& h_{3}=\left(T_{1}+T_{2}\right)-2\left(T_{0}+T_{4}\right), \\
& h_{4}=2\left(T_{4} c-T_{3}\right)+\left(T_{1}-T_{2}\right),
\end{aligned}
$$

where

$$
\begin{aligned}
& T_{0}=g_{0}^{2}, \\
& T_{1}=\left(g_{0}+g_{3}+g_{4}\right)^{2}, \\
& T_{2}=\left(g_{0}+g_{3}-g_{4}\right)^{2}, \\
& T_{3}=2 g_{3} g_{4}, \\
& T_{4}=g_{3}^{2} .
\end{aligned}
$$

Hence, the total cost is dominated by 1 squaring in $\mathbb{F}_{q^{3}}$, or by 4 squarings and 1 multiplication in $\mathbb{F}_{q}$. Note that the compression function $\mathcal{C}$ in this case cannot have an inverse as $\mathcal{C}(g)=\mathcal{C}\left(g^{q^{3}}\right)$.

5.3. $\mathbf{S Q R}_{2345}: \mathcal{F}=\left\{I, \mathcal{C}, \mathcal{S}_{I}\right\}$ with $|I|=4$.

$$
\begin{aligned}
I & =\{2,3,4,5\}, \mathcal{C}(g)=\left[g_{2}, g_{3}, g_{4}, g_{5}\right], \mathcal{S}_{I}=\left\{S_{2,1}, S_{3,4}, S_{4,5}, S_{5,1}\right\}, \\
h_{2} & =S_{2,1}=2 g_{2}+6 g_{4} g_{5} c, \\
h_{3} & =S_{3,4}=3 g_{4}^{2}+3 g_{5}^{2} c-2 g_{3}, \\
h_{4} & =S_{4,5}=3 g_{2}^{2}+3 g_{3}^{2} c-2 g_{4}, \\
h_{5} & =S_{5,1}=2 g_{5}+6 g_{2} g_{3} .
\end{aligned}
$$

The above formula is a rediscovery of the squaring formula in Theorem 3.2, where we show that $\mathcal{C}\left(g^{2}\right)$ can be computed at a cost dominated by 4 multiplications in $\mathbb{F}_{q}$. Next, we observe that $\mathcal{C}\left(g^{2}\right)$ can also be computed at a cost dominated by 2 squarings in $\mathbb{F}_{q^{2}}$. This follows because, inspired by GS-squaring [12, we can write

$$
\begin{aligned}
& h_{2}+h_{3} w=S_{2,1}+S_{3,4} w=3 w\left(g_{4}+g_{5} w\right)^{2}+2\left(g_{2}-g_{3} w\right), \\
& h_{4}+h_{5} w=S_{4,5}+S_{5,2} w=3\left(g_{2}+g_{3} w\right)^{2}-2\left(g_{4}-g_{5} w\right),
\end{aligned}
$$

which requires 2 squarings in $\mathbb{F}_{q^{2}}$.

5.4. $\mathbf{S Q R}_{0134}: \mathcal{F}=\left\{I, \mathcal{C}, \mathcal{S}_{I}\right\}$ with $|I|=4$.

$$
\begin{aligned}
I & =\{0,1,3,4\}, \mathcal{C}(g)=\left[g_{0}, g_{1}, g_{3}, g_{4}\right], \mathcal{S}_{I}=\left\{S_{0,1}, S_{1,1}, S_{3,2}, S_{4,1}\right\}, \\
h_{0} & =S_{0,1}=2 g_{0}^{2}+4 g_{3} g_{4} c-1, \\
h_{1} & =S_{1,1}=6 g_{0} g_{1}+2 g_{1}, \\
h_{3} & =S_{3,2}=4 g_{0} g_{3}+2 g_{4}^{2}, \\
h_{4} & =S_{4,1}=4 g_{0} g_{4}+2 g_{3}^{2} c .
\end{aligned}
$$

In this formula, one can compute $\left\{h_{0}, h_{3}, h_{4}\right\}$ at a cost dominated by 4 squarings and 1 multiplication in $\mathbb{F}_{q}$ (see Section 5.2 . Hence, $\left\{h_{0}, h_{1}, h_{3}, h_{4}\right\}$ can be computed at a cost dominated by 4 squarings and 2 multiplications in $\mathbb{F}_{q}$. Similarly, as in the proof of Theorem 3.1, we can show that an inverse to the compression function $\mathcal{C}$ can be given as follows.

$$
\mathcal{D}\left(\left[\tilde{g}_{0}, \tilde{g}_{1}, \tilde{g}_{3}, \tilde{g}_{4}\right]\right)=\left(\tilde{g}_{0}+\tilde{g}_{1} w\right)+\left(\tilde{g}_{2}+\tilde{g}_{3} w\right) \sigma+\left(\tilde{g}_{4}+\tilde{g}_{5} w\right) \sigma^{2},
$$


where

$$
\begin{aligned}
& \tilde{g}_{2}=\frac{\tilde{g}_{3}\left(\tilde{g}_{0}-1\right)+2 \tilde{g}_{4}^{2}}{3 \tilde{g}_{1}}, \\
& \tilde{g}_{5}=\frac{\tilde{g}_{4}\left(\tilde{g}_{0}-1\right)+2 \tilde{g}_{2}^{2} c}{3 \tilde{g}_{1} c} .
\end{aligned}
$$

If $g \in G_{\Phi_{6}(q)} \backslash\{1\}$, then $g_{1}$ can never equal zero because

$$
\operatorname{Tr}_{\mathbb{F}_{q^{6}} / \mathbb{F}_{q^{2}}}\left(g^{2}\right)=g_{0}+g_{1} w \in \mathbb{F}_{q^{2}} \backslash \mathbb{F}_{q} .
$$

Hence, the decompression function $\mathcal{D}$ is well defined and $\mathcal{D}(\mathcal{C}(g))=g$ for all $g \in G_{\Phi_{6}(q)} \backslash\{1\}$. The decompression can be performed at a cost dominated by 4 multiplications, 2 squarings and 1 inversion in $\mathbb{F}_{q}$.

5.5. Other formulae $\mathcal{F}=\left\{I, \mathcal{C}, \mathcal{S}_{I}\right\}$ with $|I|=4$. There are 4 more classes of squaring formulae with $|I|=4$ and we list one from each class that seems to be the most efficient one in its class.

$$
\begin{aligned}
I & =\{0,1,2,3\}, \mathcal{C}(g)=\left[g_{0}, g_{1}, g_{2}, g_{3}\right], \mathcal{S}_{I}=\left\{S_{0,3}, S_{1,1}, S_{2,2}, S_{3,3}\right\}, \\
h_{0} & =S_{0,3}=3 g_{0}^{2}-2 g_{0}+3 g_{1}^{2} c, \\
h_{1} & =S_{1,1}=6 g_{0} g_{1}+2 g_{1}, \\
h_{2} & =S_{2,2}=3 g_{0} g_{2}+3 g_{1} g_{3} c-g_{2}, \\
h_{3} & =S_{3,3}=3 g_{0} g_{3}+3 g_{1} g_{2}+g_{3} . \\
I & =\{0,1,4,5\}, \mathcal{C}(g)=\left[g_{0}, g_{1}, g_{4}, g_{5}\right], \mathcal{S}_{I}=\left\{S_{0,3}, S_{1,1}, S_{4,3}, S_{5,2}\right\}, \\
h_{0} & =S_{0,3}=3 g_{0}^{2}+3 g_{1}^{2} c-2 g_{0}, \\
h_{1} & =S_{1,1}=6 g_{0} g_{1}+2 g_{1}, \\
h_{4} & =S_{4,3}=3 g_{0} g_{4}+3 g_{1} g_{5} c+g_{4}, \\
h_{5} & =S_{5,2}=3 g_{0} g_{5}+3 g_{1} g_{4}-g_{5} . \\
I & =\{1,2,3,5\}, \mathcal{C}(g)=\left[g_{1}, g_{2}, g_{3}, g_{5}\right], \mathcal{S}_{I}=\left\{S_{1,5}, S_{2,1}, S_{3,1}, S_{5,1}\right\}, \\
h_{1} & =S_{1,5}=12 g_{5}^{3} c^{2}+\left(12 g_{1}^{3}+12 g_{1} g_{2} g_{5}-36 g_{2} g_{3}^{2}-24 g_{3} g_{5}\right) c+8 g_{1}, \\
h_{2} & =S_{2,1}=2 g_{2}+6 g_{4} g_{5} c, \\
h_{3} & =S_{3,1}=4 g_{1} g_{2}+2 g_{5}^{2} c, \\
h_{5} & =S_{5,1}=6 g_{2} g_{3}+2 g_{5} . \\
I & =\{1,2,4,5\}, \mathcal{C}(g)=\left[g_{1}, g_{2}, g_{4}, g_{5}\right], \mathcal{S}_{I}=\left\{S_{1,3}, S_{2,1}, S_{4,2}, S_{5,3}\right\}, \\
h_{1} & =S_{1,3}=\left(12 g_{1} g_{2} g_{5}+12 g_{1}^{3}-36 g_{4}^{2} g_{5}\right) c-24 g_{2} g_{4}+8 g_{1}+12 g_{2}^{3}, \\
h_{2} & =S_{2,1}=2 g_{2}+6 g_{4} g_{5} c, \\
h_{4} & =S_{4,2}=4 g_{1} g_{5} c+2 g_{2}^{2}, \\
h_{5} & =S_{5,3}=-12 g_{1} g_{2}^{2}+9 g_{2} g_{4}^{2}+3 g_{2} g_{5}^{2} c+2 g_{5} . \\
& =g_{0} .
\end{aligned}
$$

For each of the above 4 formulae, one can write a decompression function $\mathcal{D}$ such that $\mathcal{D}$ is well defined and $\mathcal{D}(\mathcal{C}(g))=g$ for all $g \in G_{\Phi_{6}(q)}$. Our analysis shows that in the first two squaring formulae, the decompression functions require 1 inversion in $\mathbb{F}_{q}$, and computing $\mathcal{C}\left(g^{2}\right)$ requires more than 4 multiplications in $\mathbb{F}_{q}$. Therefore, they do not seem to yield better algorithms than the squaring formula 
in Theorem 3.2, In the latter two squaring formulae, the decompression functions do not require an inversion, and they can be given as follows:

$$
\mathcal{D}\left(\left[g_{1}, g_{2}, g_{3}, g_{5}\right]\right)=\left(g_{0}+g_{1} w\right)+\left(g_{2}+g_{3} w\right) \sigma+\left(g_{4}+g_{5} w\right) \sigma^{2},
$$

where

$$
\begin{aligned}
& g_{4}=1 / 2\left(g_{2}^{2}+3 g_{3}^{2} c\right)-2 g_{1} g_{5} c, \\
& g_{0}=\left(2 g_{1}^{2}+g_{2} g_{5}-3 g_{3} g_{4}\right) c+1,
\end{aligned}
$$

and

$$
\mathcal{D}\left(\left[g_{1}, g_{2}, g_{4}, g_{5}\right]\right)=\left(g_{0}+g_{1} w\right)+\left(g_{2}+g_{3} w\right) \sigma+\left(g_{4}+g_{5} w\right) \sigma^{2},
$$

where

$$
\begin{aligned}
& g_{3}=1 / 2\left(g_{5}^{2} c+3 g_{4}^{2}\right)-2 g_{1} g_{2}, \\
& g_{0}=\left(2 g_{1}^{2}+g_{2} g_{5}-3 g_{3} g_{4}\right) c+1,
\end{aligned}
$$

respectively. The above formulae yield factor- $3 / 2$ compression for elements $g \in$ $G_{\Phi_{6}(q)}$ with the property that decompression can be performed at a cost dominated by 3 multiplications and 3 squarings in $\mathbb{F}_{q}$. However, computing $\mathcal{C}\left(g^{2}\right)$ requires more than 6 multiplications in $\mathbb{F}_{q}$. Therefore, they do not seem to yield better algorithms than GS-squaring.

5.6. Squaring formulae $\mathcal{F}=\left\{I, \mathcal{C}, \mathcal{S}_{I}\right\}$ with $|I|=5$. There are 6 classes of squaring formulae with $|I|=5$. Our analysis shows in all of these formulae, computing $\mathcal{C}\left(g^{2}\right)$ requires more than 4 multiplications in $\mathbb{F}_{q}$. Therefore, there is no hope that they would yield better exponentiation algorithms than the formula in Theorem 3.2 unless decompression can be achieved without doing an inversion in $\mathbb{F}_{q}$ (recall that the decompression function of the squaring formula in Theorem 3.2 requires an inversion in $\left.\mathbb{F}_{q}\right)$. We found one such formula, where $\mathcal{C}\left(g^{2}\right)$ can be computed at a cost dominated by 5 multiplications in $\mathbb{F}_{q}$, and the decompression can be performed at a cost dominated by 2 multiplications and 1 squaring in $\mathbb{F}_{q}$.

The formula can be seen as an extension of the squaring formula in Theorem 3.2 and it is given as follows:

$$
\begin{aligned}
I & =\{1,2,3,4,5\}, \mathcal{C}(g)=\left[g_{0}, g_{2}, g_{3}, g_{4}, g_{5}\right], \\
\mathcal{S}_{I} & =\left\{S_{1,2}, S_{2,1}, S_{3,4}, S_{4,5}, S_{5,1}\right\}, \\
h_{1} & =-g_{1}+3\left(C_{2,3,4,5}-B_{4,5}-B_{2,3} c\right), \\
h_{2} & =2\left(g_{2}+3 c B_{4,5}\right), \\
h_{3} & =3\left(A_{4,5}-(c+1) B_{4,5}\right)-2 g_{3}, \\
h_{4} & =3\left(A_{2,3}-(c+1) B_{2,3}\right)-2 g_{4}, \\
h_{5} & =2\left(g_{5}+3 B_{2,3}\right),
\end{aligned}
$$

where

$$
\begin{aligned}
A_{i, j} & =\left(g_{i}+g_{j}\right)\left(g_{i}+c g_{j}\right), \\
B_{i, j} & =g_{i} g_{j}, \\
C_{2,3,4,5} & =\left(g_{2}+g_{5}\right)\left(g_{3} c+g_{4}\right) .
\end{aligned}
$$


It is clear from Theorem 3.1 that an inverse to the compression function $\mathcal{C}$ can be given as follows:

$$
\tilde{g}_{0}=\left(2 \tilde{g}_{1}^{2}+\tilde{g}_{2} \tilde{g}_{5}-3 \tilde{g}_{3} \tilde{g}_{4}\right) c+1 .
$$

There is one class of squaring formulae with $|I|=6$ and we list below two formulae that are the most efficient ones according to our analysis; the first can be seen as an extension of the squaring formula in Theorem 3.2 , and the second one can be seen as an extension of the squaring formula in Section 5.4

5.7. A squaring formula $\mathcal{F}=\left\{I, \mathcal{C}, \mathcal{S}_{I}\right\}$ with $|I|=6$.

$$
\begin{aligned}
I & =\{0,1,2,3,4,5\}, \mathcal{C}(g)=\left[g_{0}, g_{1}, g_{2}, g_{3}, g_{4}, g_{5}\right], \\
\mathcal{S}_{I} & =\left\{S_{0,3}, S_{1,1}, S_{2,1}, S_{3,4}, S_{4,5}, S_{5,1}\right\}, \\
h_{0} & =S_{0,3}=3 g_{0}^{2}-2 g_{0}+3 g_{1}^{2}=3\left(A_{0,1}-(c+1) B_{0,1}\right)-2 g_{0}, \\
h_{1} & =S_{1,1}=6 g_{0} g_{1}+2 g_{1}=2\left(g_{1}+3 B_{0,1}\right), \\
h_{2} & =S_{2,1}=2 g_{2}+6 g_{4} g_{5}=2\left(g_{2}+3 c B_{4,5}\right), \\
h_{3} & =S_{3,4}=3 g_{4}^{2}+3 g_{5}^{2} c-2 g_{3}=3\left(A_{4,5}-(c+1) B_{4,5}\right)-2 g_{3}, \\
h_{4} & =S_{4,5}=3 g_{2}^{2}+3 g_{3}^{2} c-2 g_{4}=3\left(A_{2,3}-(c+1) B_{2,3}\right)-2 g_{4}, \\
h_{5} & =S_{5,1}=2 g_{5}+6 g_{2} g_{3}=2\left(g_{5}+3 B_{2,3}\right),
\end{aligned}
$$

where

$$
\begin{aligned}
& A_{i, j}=\left(g_{i}+g_{j}\right)\left(g_{i}+c g_{j}\right), \\
& B_{i, j}=g_{i} g_{j} .
\end{aligned}
$$

Note that $\mathcal{C}\left(g^{2}\right)$ can be computed at a cost dominated by 6 multiplications in $\mathbb{F}_{q}$, and there is no need of a decompression function. In fact, a closer look at the formulae [12] shows that the above formula is a rediscovery of GS-squaring.

5.8. $\mathbf{S Q R}_{012345}: \mathcal{F}=\left\{I, \mathcal{C}, \mathcal{S}_{I}\right\}$ with $|I|=6$.

$$
\begin{aligned}
I & =\{0,1,2,3,4,5\}, \mathcal{C}(g)=\left[g_{0}, g_{1}, g_{2}, g_{3}, g_{4}, g_{5}\right], \\
\mathcal{S}_{I} & =\left\{S_{0,1}, S_{1,1}, S_{2,1}, S_{3,2}, S_{4,1}, S_{5,1}\right\}, \\
h_{0} & =S_{0,1}=2 g_{0}^{2}+4 g_{3} g_{4} c-1=2\left(T_{0}+T_{1} c\right)-1, \\
h_{1} & =S_{1,1}=6 g_{0} g_{1}+2 g_{1}=2\left(g_{1}+3 B_{0,1}\right), \\
h_{2} & =S_{2,1}=2 g_{2}+6 g_{4} g_{5} c=2\left(g_{2}+3 c B_{4,5}\right), \\
h_{3} & =S_{3,2}=4 g_{0} g_{3}+2 g_{4}^{2}=\left(T_{1}+T_{2}\right)-2\left(T_{0}+T_{4}\right), \\
h_{4} & =S_{4,1}=4 g_{0} g_{4}+2 g_{3}^{2} c=2\left(T_{4} c-T_{3}\right)+\left(T_{1}-T_{2}\right), \\
h_{5} & =S_{5,1}=2 g_{5}+6 g_{2} g_{3}=2\left(g_{5}+3 B_{2,3}\right),
\end{aligned}
$$

where

$$
\begin{aligned}
& T_{0}=g_{0}^{2}, \\
& T_{1}=\left(g_{0}+g_{3}+g_{4}\right)^{2}, \\
& T_{2}=\left(g_{0}+g_{3}-g_{4}\right)^{2}, \\
& T_{3}=2 g_{3} g_{4}, \\
& T_{4}=g_{3}^{2}, \\
& B_{i, j}=g_{i} g_{j} .
\end{aligned}
$$


Using the above formula, $\mathcal{C}\left(g^{2}\right)$ can be computed at a cost dominated by 4 multiplications and 4 squarings in $\mathbb{F}_{q}$, and and there is no need for a decompression function.

\section{Comparisons}

In Tables 2, 3] and 4, we compare the most efficient squaring formulae in this paper with the squaring formula in [12] which is the fastest previously-known squaring formula that can be easily adapted for multi-exponentiation algorithms. We denote our squaring formula in Theorem 3.2 by $\mathrm{SQR}_{2345}$ (also see Section 5.3) as it can be written as a function of $g_{2}, g_{3}, g_{4}, g_{5}$. Similarly, we denote the squaring formulae in Sections 5.4, 5.6 and 5.8 by $\mathrm{SQR}_{0134}, \mathrm{SQR}_{12345}$ and $\mathrm{SQR}_{012345}$, respectively.

As before, we let $M_{i}$ and $S_{i}$ denote multiplication and squaring costs in $\mathbb{F}_{p^{i}}$, and assume a) $M_{2 i}=3 M_{i}, M_{3 i}=6 M_{i}$; and $\left.b\right) S_{1}=M_{1}, S_{2 i}=2 M_{i}, S_{3 i}=M_{i}+4 S_{i}$.

TABLE 2. A comparison of squaring algorithms in $G_{\Phi_{6}(q)} \subset \mathbb{F}_{q^{6}}^{*}$, where $q=p^{i} \equiv 1(\bmod 6)$ and $i=2^{a} 3^{b}$ with $a>0$.

\begin{tabular}{|l|l|l|}
\hline Algorithm & Squaring cost & Decompression cost \\
\hline \hline $\mathrm{SQR}_{2345}$ & $\left(6^{b} \cdot 3^{a} \cdot 4\right) M_{1}$ & $1 I_{i}+\left(6^{b} \cdot 3^{a} \cdot 5\right) M_{1}$ \\
\hline $\mathrm{SQR}_{0134}$ & $\left(6^{b} \cdot 3^{a} \cdot 14 / 3\right) M_{1}$ & $1 I_{i}+\left(6^{b} \cdot 3^{a} \cdot 16 / 3\right) M_{1}$ \\
\hline $\mathrm{SQR}_{12345}$ & $\left(6^{b} \cdot 3^{a} \cdot 5\right) M_{1}$ & $\left(6^{b} \cdot 3^{a} \cdot 8 / 3\right) M_{1}$ \\
\hline $\mathrm{SQR}_{012345}$ & $\left(6^{b} \cdot 3^{a} \cdot 20 / 3\right) M_{1}$ & 0 \\
\hline GS-squaring [12 & $\left(6^{b} \cdot 3^{a} \cdot 6\right) M_{1}$ & 0 \\
\hline
\end{tabular}

According to Table 2. $\mathrm{SQR}_{2345}$ is the fastest squaring algorithm. In particular, $\mathrm{SQR}_{2345}$ is $33 \%$ faster than GS-squaring. When evaluating the costs of squaring algorithms in Table 2, we assumed that $M_{2^{a} 3^{b}}=\left(6^{b} \cdot 3^{a}\right) M_{1}$ and $S_{2^{a} 3^{b}}=2 M_{2^{a-1} 3^{b}}=$ $\left(6^{b} \cdot 3^{a} \cdot 2 / 3\right) M_{1}$. In particular, the costs of $\mathrm{SQR}_{2345}$ and GS-squaring are computed as $2 S_{2^{a+1} 3^{b}}=4 M_{2^{a} 3^{b}}=\left(6^{b} \cdot 3^{a} \cdot 4\right) M_{1}$ and $3 S_{2^{a+1} 3^{b}}=6 M_{2^{a} 3^{b}}=\left(6^{b} \cdot 3^{a} \cdot 6\right) M_{1}$, respectively.

It is possible to obtain better (asymptotic) running time estimates for the algorithms listed in Table 2 because using $S_{3 i}=M_{i}+4 S_{i}$ repetitively, we can show that

$$
S_{2^{a} 3^{b}}=6^{b} \cdot 3^{a} \cdot 1 / 2 \cdot\left(1+\left(2^{b} / 3^{b+1}\right)\right) M_{1} \text { for } a, b>0 .
$$

Note that $\left(M_{2^{a} 3^{b}} / S_{2^{a} 3^{b}}\right) \rightarrow 2$ as $b \rightarrow \infty$. Then, for example, the costs of $\mathrm{SQR}_{2345}$ and GS-squaring can be estimated as $2 S_{2^{a+1} 3^{b}}=\left(6^{b} \cdot 3^{a} \cdot 3\right) M_{1}$ and $3 S_{2^{a+1} 3^{b}}=$ $\left(6^{b} \cdot 3^{a} \cdot 9 / 2\right) M_{1}$, respectively. We present an asymptotic comparison of the squaring algorithms in Table 3 . According to Table $3 \mathrm{SQR}_{2345}$ is the fastest squaring algorithm, and is $33 \%$ faster than GS-squaring.

As we noted in Section 4, the decompression costs must be considered when adapting our squaring formulae in exponentiation algorithms. However, this does not seem to be a big issue when the exponent has low Hamming weight. For example, in Section 4.1 we discuss how $\mathrm{SQR}_{2345}$ can be used to improve the efficiency of exponentiation in $G_{\Phi_{12}(p)} \subset \mathbb{F}_{p^{12}}^{*}$ and the BN pairing computations. Similarly, from Table 4 we see that $\mathrm{SQR}_{2345}$ can be used to improve the efficiency of exponentiation in $G_{\Phi_{k}(p)} \subset \mathbb{F}_{p^{k}}^{*}$ and pairing computations that use elliptic curves with embedding degree $k$, for $k=18,242$

\footnotetext{
${ }^{2}$ Families of elliptic curves with embedding degree 18 and 24 are given in [7.
} 
TABLE 3. An asymptotic comparison of squaring algorithms in $G_{\Phi_{6}(q)} \subset \mathbb{F}_{q^{6}}^{*}$, where $q=p^{i} \equiv 1(\bmod 6)$ and $i=2^{a} 3^{b}$ with $a>0$ and $b \rightarrow \infty$.

\begin{tabular}{|l|l|l|}
\hline Algorithm & Squaring cost & Decompression cost \\
\hline \hline $\mathrm{SQR}_{2345}$ & $\left(6^{b} \cdot 3^{a} \cdot 3\right) M_{1}$ & $1 I_{i}+\left(6^{b} \cdot 3^{a} \cdot 9 / 2\right) M_{1}$ \\
\hline $\mathrm{SQR}_{0134}$ & $\left(6^{b} \cdot 3^{a} \cdot 4\right) M_{1}$ & $1 I_{i}+\left(6^{b} \cdot 3^{a} \cdot 5\right) M_{1}$ \\
\hline $\mathrm{SQR}_{12345}$ & $\left(6^{b} \cdot 3^{a} \cdot 5\right) M_{1}$ & $\left(6^{b} \cdot 3^{a} \cdot 5 / 2\right) M_{1}$ \\
\hline $\mathrm{SQR}_{012345}$ & $\left(6^{b} \cdot 3^{a} \cdot 6\right) M_{1}$ & 0 \\
\hline GS-squaring [12] & $\left(6^{b} \cdot 3^{a} \cdot 9 / 2\right) M_{1}$ & 0 \\
\hline
\end{tabular}

TABLE 4. A comparison of squaring algorithms in $G_{\Phi_{6}(q)} \subset \mathbb{F}_{q^{6}}^{*}$, $q \equiv 1(\bmod 6)$.

\begin{tabular}{|c|c|c|c|c|}
\hline$q=p^{i}$ & Squaring cost & Decompression cost & Squaring cost & Decompression cost \\
\hline & \multicolumn{2}{|c|}{$\overline{\mathrm{SQR}_{2345}}$} & \multicolumn{2}{|c|}{$\overline{\mathrm{SQR}_{0134}}$} \\
\hline$i$ & $\min \left(4 M_{i}, 2 S_{2 i}\right)$ & $1 I_{i}+3 M_{i}+3 S_{i}$ & $2 M_{i}+4 S_{i}$ & $1 I_{i}+4 M_{i}+2 S_{i}$ \\
\hline$i=1$ & $4 M_{1}$ & $1 I_{1}+6 M_{1}$ & $6 M_{1}$ & $1 I_{1}+6 M_{1}$ \\
\hline$i=2$ & $12 M_{1}$ & $1 I_{2}+15 M_{1}$ & $14 M_{1}$ & $1 I_{2}+16 M_{1}$ \\
\hline$i=3$ & $22 M_{1}$ & $1 I_{3}+33 M_{1}$ & $32 M_{1}$ & $1 I_{3}+34 M_{1}$ \\
\hline \multirow[t]{2}{*}{$i=4$} & $36 M_{1}$ & $1 I_{4}+45 M_{1}$ & $42 M_{1}$ & $1 I_{4}+48 M_{1}$ \\
\hline & \multicolumn{2}{|c|}{$\mathrm{SQR}_{12345}$} & \multicolumn{2}{|c|}{$\mathrm{SQR}_{012345}$} \\
\hline$i$ & $5 M_{i}$ & $2 M_{i}+1 S_{i}$ & $4 M_{i}+4 S_{i}$ & 0 \\
\hline$i=1$ & $5 M_{1}$ & $3 M_{1}$ & $8 M_{1}$ & 0 \\
\hline$i=2$ & $15 M_{1}$ & $8 M_{1}$ & $20 M_{1}$ & 0 \\
\hline$i=3$ & $30 M_{1}$ & $17 M_{1}$ & $44 M_{1}$ & 0 \\
\hline \multirow[t]{2}{*}{$i=4$} & $45 M_{1}$ & $24 M_{1}$ & $60 M_{1}$ & 0 \\
\hline & \multicolumn{2}{|c|}{ GS-squaring [12] } & & \\
\hline$i$ & $\min \left(6 M_{i}, 3 S_{2 i}\right)$ & 0 & & \\
\hline$i=1$ & $6 M_{1}$ & 0 & & \\
\hline$i=2$ & $18 M_{1}$ & 0 & & \\
\hline$i=3$ & $33 M_{1}$ & 0 & & \\
\hline$i=4$ & $54 M_{1}$ & 0 & & \\
\hline
\end{tabular}

When the exponent is chosen at random in an exponentiation algorithm, it seems more advantageous to use either $\mathrm{SQR}_{12345}$ or GS-squaring because decompression is a relatively inexpensive operation. Let $e$ be an exponent with $\ell$-bits. The width- $w$ NAF representation of $e$ contains on average $\ell /(w+1)$ nonzero digits which determines the number of multiplications and the number of decompression operations in the exponentiation algorithm. Then, the cost of computing $g^{e} \in G_{\Phi_{6}\left(p^{i}\right)} \subset \mathbb{F}_{p^{6 i}}^{*}$ using $\mathrm{SQR}_{12345}$ and GS-squaring would be dominated by

$$
\begin{aligned}
& \operatorname{EXP}_{12345}(i, w, \ell)=(5 \ell) M_{i}+\frac{\ell}{w+1}\left(M_{6 i}\right)+\frac{\ell}{w+1}\left(2 M_{i}+1 S_{i}\right), \\
& \operatorname{EXP}_{\mathrm{GS}}(i, w, \ell)=(3 \ell)\left(S_{2 i}\right)+\frac{\ell}{w+1} M_{6 i},
\end{aligned}
$$

respectively. Table 5 compares the exponentiation costs per bit of $\ell$ for particular cases of $i$ and $w$. 
TABLE 5. A comparison of exponentiation costs in $G_{\Phi_{6}(q)} \subset \mathbb{F}_{q^{*}}$. $\exp _{12345}$ and $\exp _{\mathrm{GS}}$ are the exponentiation costs per bit of exponent, where the exponentiation is performed based on $\mathrm{SQR}_{12345}$ and GS-squaring, respectively. The exponent is represented in width- $w$ NAF and $q=p^{i} \equiv 1(\bmod 6)$.

\begin{tabular}{|l|l|l|l|l|l|l|}
\hline$(i, w)$ & $(2,2)$ & $(3,2)$ & $(4,2)$ & $(2,3)$ & $(3,3)$ & $(4,3)$ \\
\hline $\exp _{12345}$ & 35.6 & 71.6 & 107 & 30.5 & 60.75 & 91.5 \\
\hline $\exp _{\mathrm{GS}}$ & 36 & 69 & 108 & 31.5 & 60 & 94.5 \\
\hline
\end{tabular}

\section{Concluding Remarks}

We proposed new squaring formulae for cyclotomic subgroups $G_{\Phi_{6}(p)} \subset \mathbb{F}_{q^{6}}^{*}$, where $q \equiv 1(\bmod 6)$, and demonstrated that the formulae can be used to speed up cryptographic protocols. Our operation counts ignored the cost of addition, subtraction and the cost of multiplying a finite field element by a small integer. Therefore, it would be desirable to implement the algorithms to verify their relative efficiency.

\section{ACKNOWLEDGMENT}

The author would like to thank Diego F. Aranha and Alfred Menezes for fruitful discussion and for their useful comments on the paper. The author would also like to thank the two anonymous referees for their valuable suggestions.

\section{ApPendix A}

A.1. $\mathcal{H}_{0}=\left\{S_{0, j}: j=1,2, \ldots, 13\right\}$.

$S_{0,1}=2 g_{0}^{2}+4 g_{3} g_{4} c-1$,

$S_{0,2}=-6 g_{0}^{2}+4 g_{0}+12 g_{2} g_{5} c+3$,

$S_{0,3}=3 g_{0}^{2}-2 g_{0}+3 g_{1}^{2} c$,

$S_{0,4}=1+\left(2 g_{1}^{2}+4 g_{2} g_{5}\right) c$,

$S_{0,5}=\left(3 g_{2} g_{5}+3 g_{3} g_{4}\right) c+g_{0}$,

$S_{0,6}=\left(-6 g_{1}^{2}+12 g_{3} g_{4}\right) c-3+4 g_{0}$,

$S_{0,7}=27 g_{1}^{2} g_{3}^{3} c^{3}+\left(\left(-27 g_{1} g_{2} g_{3}^{2}-72 g_{1}^{4}\right) g_{0}+81 g_{1}^{2} g_{2}^{2} g_{3}-48 g_{1}^{4}\right.$

$\left.+27 g_{1} g_{2} g_{3}^{2}+18 g_{3}^{3}\right) c^{2}+\left(\left(-9 g_{1} g_{2}^{3}-42 g_{1}^{2}\right) g_{0}-36 g_{1}^{2}\right.$

$\left.+6 g_{2}^{2} g_{3}+21 g_{1} g_{2}^{3}\right) c-3+4 g_{0}$,

$S_{0,8}=-3 / 2 g_{1} g_{5}^{3} c^{3}+\left(-9 / 2 g_{0} g_{4} g_{5}^{2}-12 g_{1}^{4}+21 / 2 g_{4} g_{5}^{2}+63 / 2 g_{1} g_{4}^{2} g_{5}\right) c^{2}$

$+\left(\left(12 g_{1}^{2}-27 / 2 g_{4}^{3}\right) g_{0}+27 / 2 g_{4}^{3}-9 g_{1}^{2}\right) c-3+4 g_{0}$,

$S_{0,9}=\left(63 / 2 g_{1} g_{2} g_{3}^{2}-12 g_{1}^{4}+27 / 2 g_{3}^{3}-27 / 2 g_{0} g_{3}^{3}\right) c^{2}$

$+\left(\left(-9 / 2 g_{2}^{2} g_{3}+12 g_{1}^{2}\right) g_{0}-3 / 2 g_{1} g_{2}^{3}+21 / 2 g_{2}^{2} g_{3}-9 g_{1}^{2}\right) c$

$-3+4 g_{0}$,

$S_{0,10}=-729 / 4 g_{1}^{2} g_{3}^{6} c^{5}+\left(324 g_{1}^{4} g_{3}^{3}+243 / 4 g_{1} g_{2} g_{3}^{5}-243 / 2 g_{1}^{2} g_{2}^{2} g_{3}^{4}\right.$

$\left.+486 g_{0} g_{1}^{4} g_{3}^{3}+243 / 16 g_{3}^{6}-162 g_{1}^{5} g_{2} g_{3}^{2}\right) c^{4}$

$+\left(81 g_{0} g_{1}^{2} g_{3}^{3}+144 g_{1}^{6}+54 g_{1}^{4} g_{2}^{2} g_{3}-513 g_{1}^{3} g_{2} g_{3}^{2}\right.$ 


$$
\begin{aligned}
& \left.+\left(-81 / 4 g_{2}^{4} g_{3}^{2}-297 / 2 g_{3}^{3}\right) g_{1}^{2}+81 / 2 g_{1} g_{2}^{3} g_{3}^{3}+81 / 8 g_{2}^{2} g_{3}^{4}\right) c^{3} \\
& +\left(\left(-144 g_{1}^{4}+27 / 8 g_{3}^{3}\right) g_{0}+132 g_{1}^{4}+18 g_{1}^{3} g_{2}^{3}-225 / 2 g_{1}^{2} g_{2}^{2} g_{3}\right. \\
& \left.+\left(27 / 4 g_{2}^{5} g_{3}+207 / 8 g_{2} g_{3}^{2}\right) g_{1}+27 / 16 g_{2}^{4} g_{3}^{2}+117 / 8 g_{3}^{3}\right) c^{2} \\
& +\left(6 g_{2}^{2} g_{3}-96 g_{0} g_{1}^{2}+12 g_{1} g_{2}^{3}+18 g_{1}^{2}\right) c-3+4 g_{0}, \\
& S_{0,11}=\left(27 / 4 g_{1} g_{4} g_{5}^{5}-81 / 4 g_{1}^{2} g_{4}^{2} g_{5}^{4}\right) c^{5} \\
& +\left(-162 g_{1}^{5} g_{4}^{2} g_{5}+54 g_{1}^{4} g_{4} g_{5}^{2}-243 / 2 g_{1}^{2} g_{4}^{4} g_{5}^{2}\right. \\
& \left.+27 / 16 g_{4}^{2} g_{5}^{4}+81 / 2 g_{1} g_{4}^{3} g_{5}^{3}+18 g_{1}^{3} g_{5}^{3}\right) c^{4} \\
& +\left(486 g_{0} g_{1}^{4} g_{4}^{3}+144 g_{1}^{6}+324 g_{1}^{4} g_{4}^{3}-513 g_{1}^{3} g_{4}^{2} g_{5}\right. \\
& +\left(-225 / 2 g_{4} g_{5}^{2}-729 / 4 g_{4}^{6}\right) g_{1}^{2}+\left(243 / 4 g_{4}^{5} g_{5}+12 g_{5}^{3}\right) g_{1} \\
& \left.+81 / 8 g_{4}^{4} g_{5}^{2}\right) c^{3}+\left(\left(-144 g_{1}^{4}+81 g_{1}^{2} g_{4}^{3}\right) g_{0}+207 / 8 g_{1} g_{4}^{2} g_{5}\right. \\
& \left.+243 / 16 g_{4}^{6}-297 / 2 g_{1}^{2} g_{4}^{3}+6 g_{4} g_{5}^{2}+132 g_{1}^{4}\right) c^{2} \\
& +\left(\left(-96 g_{1}^{2}+27 / 8 g_{4}^{3}\right) g_{0}+117 / 8 g_{4}^{3}+18 g_{1}^{2}\right) c-3+4 g_{0}, \\
& S_{0,12}=\left(-729 / 2 g_{1}^{3} g_{2} g_{3}^{5}+972 g_{1}^{6} g_{3}^{3}\right) c^{5} \\
& +\left(1620 g_{1}^{5} g_{2} g_{3}^{2}-243 g_{1}^{3} g_{2}^{3} g_{3}^{3}+2592 g_{1}^{6} g_{2}^{2} g_{3}-2592 g_{0} g_{1}^{8}\right. \\
& \left.+243 / 8 g_{1} g_{2} g_{3}^{5}+243 / 2 g_{1}^{2} g_{2}^{2} g_{3}^{4}+486 g_{1}^{4} g_{3}^{3}-1728 g_{1}^{8}\right) c^{4} \\
& +\left(\left(-1080 g_{1}^{6}-324 g_{1}^{5} g_{2}^{3}\right) g_{0}-1080 g_{1}^{6}+864 g_{1}^{5} g_{2}^{3}-540 g_{1}^{4} g_{2}^{2} g_{3}\right. \\
& +\left(-81 / 2 g_{2}^{5} g_{3}-243 g_{2} g_{3}^{2}\right) g_{1}^{3} \\
& \left.+\left(-621 / 4 g_{3}^{3}+81 g_{2}^{4} g_{3}^{2}\right) g_{1}^{2}+81 / 4 g_{1} g_{2}^{3} g_{3}^{3}\right) c^{3} \\
& +\left(\left(-54 g_{1}^{3} g_{2}^{3}+522 g_{1}^{4}\right) g_{0}+168 g_{1}^{4}-135 g_{1}^{3} g_{2}^{3}\right. \\
& \left.+\left(27 / 2 g_{2}^{6}-54 g_{2}^{2} g_{3}\right) g_{1}^{2}+\left(27 g_{2} g_{3}^{2}+27 / 8 g_{2}^{5} g_{3}\right) g_{1}+18 g_{3}^{3}\right) c^{2} \\
& +\left(\left(-165 / 2 g_{1}^{2}-9 / 4 g_{1} g_{2}^{3}\right) g_{0}+57 / 4 g_{1} g_{2}^{3}+6 g_{2}^{2} g_{3}+9 / 2 g_{1}^{2}\right) c \\
& -3+4 g_{0} \text {, } \\
& S_{0,13}=\left(-81 / 2 g_{1}^{3} g_{4} g_{5}^{5}+27 / 2 g_{1}^{2} g_{5}^{6}\right) c^{6}+\left(81 g_{1}^{2} g_{4}^{2} g_{5}^{4}+27 / 8 g_{1} g_{4} g_{5}^{5}\right. \\
& \left.-324 g_{0} g_{1}^{5} g_{5}^{3}-243 g_{1}^{3} g_{4}^{3} g_{5}^{3}+2592 g_{1}^{6} g_{4} g_{5}^{2}+864 g_{1}^{5} g_{5}^{3}\right) c^{5} \\
& +\left(\left(-54 g_{1}^{3} g_{5}^{3}-2592 g_{1}^{8}\right) g_{0}-1728 g_{1}^{8}+972 g_{1}^{6} g_{4}^{3}+1620 g_{1}^{5} g_{4}^{2} g_{5}\right. \\
& -540 g_{1}^{4} g_{4} g_{5}^{2}+\left(-135 g_{5}^{3}-729 / 2 g_{4}^{5} g_{5}\right) g_{1}^{3}+243 / 2 g_{1}^{2} g_{4}^{4} g_{5}^{2} \\
& \left.+81 / 4 g_{1} g_{4}^{3} g_{5}^{3}\right) c^{4}+\left(\left(-9 / 4 g_{1} g_{5}^{3}-1080 g_{1}^{6}\right) g_{0}-1080 g_{1}^{6}+486 g_{1}^{4} g_{4}^{3}\right. \\
& \left.-243 g_{1}^{3} g_{4}^{2} g_{5}-54 g_{1}^{2} g_{4} g_{5}^{2}+\left(243 / 8 g_{4}^{5} g_{5}+57 / 4 g_{5}^{3}\right) g_{1}\right) c^{3} \\
& +\left(27 g_{1} g_{4}^{2} g_{5}+6 g_{4} g_{5}^{2}+168 g_{1}^{4}+522 g_{0} g_{1}^{4}-621 / 4 g_{1}^{2} g_{4}^{3}\right) c^{2} \\
& +\left(-165 / 2 g_{0} g_{1}^{2}+18 g_{4}^{3}+9 / 2 g_{1}^{2}\right) c-3+4 g_{0} \text {. }
\end{aligned}
$$

A.2. $\mathcal{H}_{1}=\left\{S_{1, j}: j=1,2, \ldots, 14\right\}$.

$$
\begin{aligned}
& S_{1,1}=6 g_{0} g_{1}+2 g_{1}, \\
& S_{1,2}=-g_{1}+3 g_{2} g_{4}+3 g_{3} g_{5} c, \\
& S_{1,3}=\left(12 g_{1} g_{2} g_{5}+12 g_{1}^{3}-36 g_{4}^{2} g_{5}\right) c-24 g_{2} g_{4}+8 g_{1}+12 g_{2}^{3}, \\
& S_{1,4}=\left(-36 g_{1} g_{3} g_{4}+12 g_{1}^{3}+36 g_{4}^{2} g_{5}\right) c+24 g_{2} g_{4}+8 g_{1}-12 g_{2}^{3}, \\
& S_{1,5}=12 g_{5}^{3} c^{2}+\left(12 g_{1}^{3}+12 g_{1} g_{2} g_{5}-36 g_{2} g_{3}^{2}-24 g_{3} g_{5}\right) c+8 g_{1}, \\
& S_{1,6}=3 / 2 g_{5}^{3} c^{2}+\left(-6 g_{1} g_{2} g_{5}+9 / 2 g_{4}^{2} g_{5}\right) c-g_{1}+3 g_{2} g_{4}, \\
& S_{1,7}=4 / 3 g_{5}^{3} c^{2}+\left(4 / 3 g_{1}^{3}-4 g_{1} g_{2} g_{5}\right) c+4 / 3 g_{2}^{3},
\end{aligned}
$$




$$
\begin{aligned}
S_{1,8}= & \left(-6 g_{1} g_{2} g_{5}+9 / 2 g_{2} g_{3}^{2}+3 g_{3} g_{5}\right) c-g_{1}+3 / 2 g_{2}^{3}, \\
S_{1,9}= & \left(12 g_{1}^{3}+9 / 2 g_{2} g_{3}^{2}-18 g_{1} g_{3} g_{4}\right) c-3 g_{2} g_{4}+8 g_{1}+3 / 2 g_{2}^{3}, \\
S_{1,10}= & 12 g_{5}^{3} c^{2}+\left(-18 g_{0} g_{3} g_{5}+9 / 2 g_{2} g_{3}^{2}-3 g_{3} g_{5}\right) c-g_{1}+3 / 2 g_{2}^{3}, \\
S_{1,11}= & 3 / 2 g_{5}^{3} c^{2}+\left(12 g_{1}^{3}-18 g_{1} g_{3} g_{4}-3 g_{3} g_{5}+9 / 2 g_{4}^{2} g_{5}\right) c+8 g_{1}, \\
S_{1,12}= & -18 g_{0} g_{2} g_{4}-g_{1}+12 g_{2}^{3}-3 g_{2} g_{4}+9 / 2 g_{4}^{2} g_{5} c+3 / 2 g_{5}^{3} c^{2}, \\
S_{1,13}= & \left(-9 / 2 g_{1} g_{4} g_{5}^{2}+3 / 4 g_{5}^{3}\right) c^{2} \\
& +\left(-27 / 2 g_{1} g_{4}^{3}+6 g_{1}^{3}+18 g_{1}^{2} g_{2} g_{4}+9 / 4 g_{4}^{2} g_{5}\right) c \\
& +7 / 2 g_{1}+3 / 2 g_{2} g_{4}, \\
S_{1,14}= & \left(18 g_{1}^{2} g_{3} g_{5}-27 / 2 g_{1} g_{3}^{3}\right) c^{2} \\
& +\left(-9 / 2 g_{1} g_{2}^{2} g_{3}+3 / 2 g_{3} g_{5}+6 g_{1}^{3}+9 / 4 g_{2} g_{3}^{2}\right) c \\
& +7 / 2 g_{1}+3 / 4 g_{2}^{3} .
\end{aligned}
$$

A.3. $\mathcal{H}_{2}=\left\{S_{2, j}: j=1,2, \ldots, 7\right\}$.

$$
\begin{aligned}
S_{2,1}= & 2 g_{2}+6 g_{4} g_{5} c \\
S_{2,2}= & 3 g_{0} g_{2}+3 g_{1} g_{3} c-g_{2}, \\
S_{2,3}= & \left(-12 g_{1} g_{5}^{2}+9 g_{3}^{2} g_{5}\right) c^{2}+3 g_{2}^{2} g_{5} c+2 g_{2}, \\
S_{2,4}= & \left(3 g_{1} g_{3}+3 g_{2}^{2} g_{5}-9 g_{2} g_{3} g_{4}+6 g_{1}^{2} g_{2}\right) c+2 g_{2}, \\
S_{2,5}= & \left(18 g_{1} g_{2} g_{3} g_{5}-27 / 2 g_{2} g_{3}^{3}\right) c^{2} \\
& +\left(6 g_{1}^{2} g_{2}-9 / 2 g_{2}^{3} g_{3}+3 g_{2}^{2} g_{5}+3 g_{1} g_{3}\right) c+2 g_{2}, \\
S_{2,6}= & \left(81 / 2 g_{1} g_{3}^{4}-54 g_{1}^{2} g_{3}^{2} g_{5}\right) c^{3} \\
& +\left(-18 g_{1}^{3} g_{3}-27 / 4 g_{2} g_{3}^{3}+9 / 2 g_{3}^{2} g_{5}+27 / 2 g_{1} g_{2}^{2} g_{3}^{2}\right) c^{2} \\
& +\left(-9 / 4 g_{2}^{3} g_{3}+3 g_{2}^{2} g_{5}+6 g_{1}^{2} g_{2}-21 / 2 g_{1} g_{3}\right) c+2 g_{2},
\end{aligned}
$$

$S_{2,7}=2187 / 8 g_{3}^{8} g_{5} c^{6}$

$+\left(243 g_{2}^{2} g_{3}^{6} g_{5}-2187 / 2 g_{1}^{2} g_{2} g_{3}^{6}-8019 / 8 g_{1} g_{3}^{7}+486 g_{1}^{5} g_{3}^{4}\right) c^{5}$

$+\left(-4293 / 4 g_{1} g_{2}^{2} g_{3}^{5}-81 g_{1}^{4} g_{2} g_{3}^{3}+1215 / 8 g_{2} g_{3}^{6}-243 / 2 g_{1}^{2} g_{2}^{3} g_{3}^{4}\right.$

$\left.+567 / 8 g_{2}^{4} g_{3}^{4} g_{5}+108 g_{1}^{5} g_{2}^{2} g_{3}^{2}+1539 / 2 g_{1}^{3} g_{3}^{4}-891 / 4 g_{3}^{5} g_{5}\right) c^{4}$

$+\left(-216 g_{1}^{5} g_{3}-18 g_{1}^{4} g_{2}^{3} g_{3}+198 g_{1}^{3} g_{2}^{2} g_{3}^{2}\right.$

$+\left(27 g_{2}^{5} g_{3}^{2}+1377 / 4 g_{2} g_{3}^{3}\right) g_{1}^{2}+\left(2997 / 4 g_{3}^{4}-1593 / 8 g_{2}^{4} g_{3}^{3}\right) g_{1}$

$\left.+27 / 4 g_{2}^{6} g_{3}^{2} g_{5}-603 / 4 g_{2}^{2} g_{3}^{3} g_{5}+54 g_{2}^{3} g_{3}^{4}\right) c^{3}$

$+\left(\left(6 g_{2}^{4}-342 g_{3}\right) g_{1}^{3}-189 / 2 g_{1}^{2} g_{2}^{3} g_{3}\right.$

$+\left(6 g_{2}^{5} g_{5}+2241 / 4 g_{2}^{2} g_{3}^{2}-9 / 4 g_{2}^{6} g_{3}\right) g_{1}$

$\left.-57 / 2 g_{2}^{4} g_{3} g_{5}+45 g_{3}^{2} g_{5}-54 g_{2} g_{3}^{3}+27 / 8 g_{2}^{5} g_{3}^{2}\right) c^{2}$

$+\left(6 g_{1}^{2} g_{2}+\left(-132 g_{3}+9 / 2 g_{2}^{4}\right) g_{1}+18 g_{2}^{3} g_{3}-3 / 4 g_{2}^{7}+3 g_{2}^{2} g_{5}\right) c+2 g_{2}$.

A.4. $\mathcal{H}_{3}=\left\{S_{3, j}: j=1,2, \ldots, 10\right\}$.

$$
\begin{aligned}
& S_{3,1}=4 g_{1} g_{2}+2 g_{5}^{2} c, \\
& S_{3,2}=4 g_{0} g_{3}+2 g_{4}^{2}, \\
& S_{3,3}=3 g_{0} g_{3}+3 g_{1} g_{2}+g_{3}, \\
& S_{3,4}=-2 g_{3}+3 g_{4}^{2}+3 g_{5}^{2} c, \\
& S_{3,5}=12 g_{0} g_{3}+4 g_{3}-6 g_{5}^{2} c,
\end{aligned}
$$




$$
\begin{aligned}
S_{3,6}= & 12 g_{1} g_{2}+4 g_{3}-6 g_{4}^{2}, \\
S_{3,7}= & \left(-27 / 2 g_{3}^{4}+18 g_{1} g_{3}^{2} g_{5}\right) c^{2}+\left(6 g_{1}^{2} g_{3}-9 / 2 g_{2}^{2} g_{3}^{2}+3 g_{2} g_{3} g_{5}\right) c \\
& +3 g_{1} g_{2}+4 g_{3} \\
S_{3,8}= & \left(6 g_{1}^{2} g_{3}+9 / 2 g_{2}^{2} g_{3}^{2}-18 g_{1} g_{2} g_{3} g_{4}-9 g_{3}^{2} g_{4}+12 g_{1}^{3} g_{2}\right) c \\
& +3 / 2 g_{2}^{4}-6 g_{2}^{2} g_{4}+12 g_{1} g_{2}+4 g_{3}, \\
S_{3,9}= & \left(54 g_{1}^{2} g_{3}^{2} g_{4}-27 / 2 g_{1} g_{2} g_{3}^{3}+27 / 4 g_{3}^{4}-36 g_{1}^{4} g_{3}\right) c^{2} \\
& +\left(-9 / 2 g_{1} g_{2}^{3} g_{3}+12 g_{1}^{3} g_{2}-21 g_{1}^{2} g_{3}+27 / 4 g_{2}^{2} g_{3}^{2}-27 / 2 g_{3}^{2} g_{4}\right) c \\
& +3 / 2 g_{2}^{4}-6 g_{2}^{2} g_{4}+12 g_{1} g_{2}+4 g_{3}, \\
S_{3,10} & \left.+729 / 4 g_{1}^{2} g_{3}^{7}-2187 / 8 g_{3}^{8} g_{4}\right) c^{5} \\
& +\left(-243 g_{2}^{2} g_{3}^{6} g_{4}+81 g_{1}^{4} g_{3}^{4}-243 / 8 g_{3}^{7}+1053 / 2 g_{1}^{2} g_{2}^{2} g_{3}^{5}\right. \\
& \left.+243 / 2 g_{1} g_{2} g_{3}^{6}-162 g_{1}^{5} g_{2} g_{3}^{3}\right) c^{4} \\
& +\left(-36 g_{1}^{5} g_{2}^{3} g_{3}+72 g_{1}^{4} g_{2}^{2} g_{3}^{2}-567 / 2 g_{1}^{3} g_{2} g_{3}^{3}\right. \\
& +\left(351 / 4 g_{2}^{4} g_{3}^{3}-81 / 4 g_{3}^{4}\right) g_{1}^{2}+999 / 4 g_{1} g_{2}^{3} g_{3}^{4} \\
& \left.-567 / 8 g_{2}^{4} g_{3}^{4} g_{4}-513 / 4 g_{2}^{2} g_{3}^{5}+891 / 4 g_{3}^{5} g_{4}\right) c^{3} \\
& +\left(\left(12 g_{2}^{4}-36 g_{3}\right) g_{1}^{4}-81 g_{1}^{3} g_{2}^{3} g_{3}+\left(-9 / 2 g_{2}^{6} g_{3}+27 g_{2}^{2} g_{3}^{2}\right) g_{1}^{2}\right. \\
& +\left(-27 g_{2} g_{3}^{3}+225 / 4 g_{2}^{5} g_{3}^{2}\right) g_{1}-459 / 8 g_{2}^{4} g_{3}^{3} \\
& \left.+603 / 4 g_{2}^{2} g_{3}^{3} g_{4}-27 / 4 g_{2}^{6} g_{3}^{2} g_{4}+27 / 2 g_{3}^{4}\right) c^{2} \\
& +\left(12 g_{1}^{3} g_{2}+\left(-21 g_{3}+9 g_{2}^{4}\right) g_{1}^{2}+\left(-45 g_{2}^{3} g_{3}+3 / 2 g_{2}^{7}-6 g_{2}^{5} g_{4}\right) g_{1}\right. \\
& \left.+27 g_{2}^{2} g_{3}^{2}-54 g_{3}^{2} g_{4}+57 / 2 g_{2}^{4} g_{3} g_{4}-15 / 2 g_{2}^{6} g_{3}\right) c \\
& +3 / 2 g_{2}^{4}-6 g_{2}^{2} g_{4}+12 g_{1} g_{2}+4 g_{3} . \\
& +1019
\end{aligned}
$$

A.5. $\mathcal{H}_{4}=\left\{S_{4, j}: j=1,2, \ldots, 10\right\}$.

$S_{4,1}=4 g_{0} g_{4}+2 g_{3}^{2} c$,

$S_{4,2}=4 g_{1} g_{5} c+2 g_{2}^{2}$,

$S_{4,3}=3 g_{0} g_{4}+3 g_{1} g_{5} c+g_{4}$,

$S_{4,4}=12 g_{0} g_{4}-6 g_{2}^{2}+4 g_{4}$,

$S_{4,5}=3 g_{2}^{2}+3 g_{3}^{2} c-2 g_{4}$,

$S_{4,6}=\left(12 g_{1} g_{5}-6 g_{3}^{2}\right) c+4 g_{4}$,

$S_{4,7}=-9 / 2 g_{4}^{2} g_{5}^{2} c^{2}+\left(6 g_{1}^{2} g_{4}+\left(3 g_{5}+18 g_{2} g_{4}^{2}\right) g_{1}\right.$

$\left.+3 g_{2} g_{4} g_{5}-27 / 2 g_{4}^{4}\right) c+4 g_{4}$,

$S_{4,8}=3 / 2 g_{5}^{4} c^{3}+\left(-18 g_{1} g_{3} g_{4} g_{5}+12 g_{1}^{3} g_{5}-6 g_{3} g_{5}^{2}+9 / 2 g_{4}^{2} g_{5}^{2}\right) c^{2}$

$+\left(-9 g_{3} g_{4}^{2}+6 g_{1}^{2} g_{4}+12 g_{1} g_{5}\right) c+4 g_{4}$,

$S_{4,9}=\left(-9 / 2 g_{1} g_{4} g_{5}^{3}+3 / 2 g_{5}^{4}\right) c^{3}$

$+\left(-36 g_{1}^{4} g_{4}-27 / 2 g_{1} g_{4}^{3} g_{5}+12 g_{1}^{3} g_{5}\right.$

$\left.+54 g_{1}^{2} g_{3} g_{4}^{2}-6 g_{3} g_{5}^{2}+27 / 4 g_{4}^{2} g_{5}^{2}\right) c^{2}$

$+\left(27 / 4 g_{4}^{4}+12 g_{1} g_{5}-21 g_{1}^{2} g_{4}-27 / 2 g_{3} g_{4}^{2}\right) c+4 g_{4}$,

$S_{4,10}=\left(-9 / 2 g_{1}^{2} g_{4} g_{5}^{6}-27 / 4 g_{3} g_{4}^{2} g_{5}^{6}+3 / 2 g_{1} g_{5}^{7}\right) c^{6}$

$+\left(-36 g_{1}^{5} g_{4} g_{5}^{3}+12 g_{1}^{4} g_{5}^{4}+351 / 4 g_{1}^{2} g_{4}^{3} g_{5}^{4}\right.$

$\left.+\left(-6 g_{3} g_{5}^{5}+225 / 4 g_{4}^{2} g_{5}^{5}\right) g_{1}-567 / 8 g_{3} g_{4}^{4} g_{5}^{4}-15 / 2 g_{4} g_{5}^{6}\right) c^{5}$ 


$$
\begin{aligned}
& +\left(-162 g_{1}^{5} g_{4}^{3} g_{5}+72 g_{1}^{4} g_{4}^{2} g_{5}^{2}-81 g_{1}^{3} g_{4} g_{5}^{3}\right. \\
& +\left(9 g_{5}^{4}+1053 / 2 g_{4}^{5} g_{5}^{2}\right) g_{1}^{2}+999 / 4 g_{1} g_{4}^{4} g_{5}^{3} \\
& \left.+\left(57 / 2 g_{4} g_{5}^{4}-243 g_{4}^{6} g_{5}^{2}\right) g_{3}-459 / 8 g_{4}^{3} g_{5}^{4}\right) c^{4} \\
& +\left(81 g_{1}^{4} g_{4}^{4}-567 / 2 g_{1}^{3} g_{4}^{3} g_{5}+\left(729 / 4 g_{4}^{7}+27 g_{4}^{2} g_{5}^{2}\right) g_{1}^{2}\right. \\
& +\left(-45 g_{4} g_{5}^{3}+243 / 2 g_{4}^{6} g_{5}\right) g_{1}+\left(-2187 / 8 g_{4}^{8}+603 / 4 g_{4}^{3} g_{5}^{2}\right) g_{3} \\
& \left.+3 / 2 g_{5}^{4}-513 / 4 g_{4}^{5} g_{5}^{2}\right) c^{3}+\left(-36 g_{1}^{4} g_{4}+12 g_{1}^{3} g_{5}\right. \\
& -81 / 4 g_{1}^{2} g_{4}^{4}-27 g_{1} g_{4}^{3} g_{5}+\left(-6 g_{5}^{2}+891 / 4 g_{4}^{5}\right) g_{3} \\
& \left.+27 g_{4}^{2} g_{5}^{2}-243 / 8 g_{4}^{7}\right) c^{2}+\left(12 g_{1} g_{5}+27 / 2 g_{4}^{4}-21 g_{1}^{2} g_{4}-54 g_{3} g_{4}^{2}\right) c \\
& +4 g_{4} .
\end{aligned}
$$

A.6. $\mathcal{H}_{5}=\left\{S_{5, j}: j=1,2, \ldots, 7\right\}$.

$$
\begin{aligned}
S_{5,1}= & 6 g_{2} g_{3}+2 g_{5}, \\
S_{5,2}= & 3 g_{0} g_{5}+3 g_{1} g_{4}-g_{5}, \\
S_{5,3}= & -12 g_{1} g_{2}^{2}+9 g_{2} g_{4}^{2}+3 g_{2} g_{5}^{2} c+2 g_{5}, \\
S_{5,4}= & \left(3 g_{2} g_{5}^{2}-9 g_{3} g_{4} g_{5}+6 g_{1}^{2} g_{5}\right) c+2 g_{5}+3 g_{1} g_{4}, \\
S_{5,5}= & -9 / 2 g_{4} g_{5}^{3} c^{2}+\left(6 g_{1}^{2} g_{5}+18 g_{1} g_{2} g_{4} g_{5}+3 g_{2} g_{5}^{2}-27 / 2 g_{4}^{3} g_{5}\right) c \\
& +2 g_{5}+3 g_{1} g_{4}, \\
S_{5,6}= & \left(27 / 2 g_{1} g_{4}^{2} g_{5}^{2}-9 / 4 g_{4} g_{5}^{3}\right) c^{2} \\
& +\left(-18 g_{1}^{3} g_{4}+\left(-54 g_{2} g_{4}^{2}+6 g_{5}\right) g_{1}^{2}+81 / 2 g_{1} g_{4}^{4}+3 g_{2} g_{5}^{2}-27 / 4 g_{4}^{3} g_{5}\right) c \\
& -21 / 2 g_{1} g_{4}+9 / 2 g_{2} g_{4}^{2}+2 g_{5}, \\
S_{5,7}= & \left.27 / 4 g_{2} g_{4}^{2} g_{5}^{6}+27 g_{1}^{2} g_{4}^{2} g_{5}^{5}-3 / 4 g_{5}^{7}-9 / 4 g_{1} g_{4} g_{5}^{6}\right) c^{5} \\
& +\left(108 g_{1}^{5} g_{4}^{2} g_{5}^{2}-18 g_{1}^{4} g_{4} g_{5}^{3}+6 g_{1}^{3} g_{5}^{4}-243 / 2 g_{1}^{2} g_{4}^{4} g_{5}^{3}\right. \\
& \left.+\left(-1593 / 8 g_{4}^{3} g_{5}^{4}+6 g_{2} g_{5}^{5}\right) g_{1}+27 / 8 g_{4}^{2} g_{5}^{5}+567 / 8 g_{2} g_{4}^{4} g_{5}^{4}\right) c^{4} \\
& +\left(486 g_{1}^{5} g_{4}^{4}-81 g_{1}^{4} g_{4}^{3} g_{5}+198 g_{1}^{3} g_{4}^{2} g_{5}^{2}\right. \\
& +\left(-2187 / 2 g_{4}^{6} g_{5}-189 / 2 g_{4} g_{5}^{3}\right) g_{1}^{2}+\left(9 / 2 g_{5}^{4}-4293 / 4 g_{4}^{5} g_{5}^{2}\right) g_{1} \\
& \left.+\left(-57 / 2 g_{4} g_{5}^{4}+243 g_{4}^{6} g_{5}^{2}\right) g_{2}+54 g_{4}^{4} g_{5}^{3}\right) c^{3} \\
& +\left(-216 g_{1}^{5} g_{4}+1539 / 2 g_{1}^{3} g_{4}^{4}+1377 / 4 g_{1}^{2} g_{4}^{3} g_{5}\right. \\
& +\left(2241 / 4 g_{4}^{2} g_{5}^{2}-8019 / 8 g_{4}^{7}\right) g_{1}+\left(-603 / 4 g_{4}^{3} g_{5}^{2}+2187 / 8 g_{4}^{8}\right) g_{2} \\
& \left.+18 g_{4} g_{5}^{3}+1215 / 8 g_{4}^{6} g_{5}\right) c^{2}+\left(-342 g_{1}^{3} g_{4}+6 g_{1}^{2} g_{5}+2997 / 4 g_{1} g_{4}^{4}\right. \\
& \left.+\left(3 g_{5}^{2}-891 / 4 g_{4}^{5}\right) g_{2}-54 g_{4}^{3} g_{5}\right) c+2 g_{5}-132 g_{1} g_{4}+45 g_{2} g_{4}^{2} . \\
&
\end{aligned}
$$

\section{REFERENCES}

1. D. Aranha, K. Karabina, P. Longa, C. Gebotys, and J. López, Faster explicit formulas for computing pairings over ordinary curves, Advances in Cryptology - Eurocrypt 2011, Lecture Notes in Computer Science 6632 (2011), 48-68.

2. P. Barreto and M. Naehrig, Pairing-friendly elliptic curves of prime order, Selected Areas in Cryptography - SAC 2005, Lecture Notes in Computer Science 3897 (2006), 319-331. MR2241646 (2007d:94026)

3. N. Benger and M. Scott, Constructing tower extensions of finite fields for implementation of pairing-based cryptography, Arithmetic of Finite Fields - WAIFI 2010, Lecture Notes in Computer Science 6087 (2010), 180-195. MR2674223 
4. J. Beuchat, J. Díaz, S. Mitsunari, E. Okamoto, F. Rodríguez-Henríquez, and T. Teruya, High-speed software implementation of the optimal Ate pairing over Barreto-Naehrig curves, Pairing-Based Cryptography - Pairing 2010, Lecture Notes in Computer Science 6487 (2010), 21-39.

5. A. Brouwer, R. Pellikaan, and E. Verheul, Doing more with fewer bits, Advances in Cryptology - ASIACRYPT '99, Lecture Notes in Computer Science 1716 (1999), 321-332.

6. J. Chung and M. Hasan, Asymmetric squaring formulae, 18th IEEE Symposium on Computer Arithmetic - ARITH 2007 (2007), 113-122.

7. D. Freeman, M. Scott, and E. Teske, A taxonomy of pairing-friendly elliptic curves, Journal of Cryptology 23 (2010), 224-280. MR2578668 (2011a:11112)

8. K. Giuliani and G. Gong, Analogues to the Gong-Harn and XTR cryptosystems, Technical Report CORR 2003-34, University of Waterloo (2003), Available at http://www.cacr.math. uwaterloo.ca/techreports/2003/corr2003-34.ps.

9. G. Gong and L. Harn, Public-key cryptosystems based on cubic finite field extensions, IEEE Transactions on Information Theory 45 (1999), 2601-2605. MR.1725159

10. R. Granger, D. Page, and N. Smart, High security pairing-based cryptography revisited, Curves over Finite Fields and Applications - Algorithmic Number Theory, Lecture Notes in Computer Science 4076 (2006), 480-494. MR2282944(2008a:94118)

11. R. Granger, D. Page, and M. Stam, A comparison of CEILIDH and XTR, Algorithmic Number Theory symposium - ANTS VI, Lecture Notes in Computer Science 3076 (2004), 235-249. MR2137357(2006a:94035)

12. R. Granger and M. Scott, Faster squaring in the cyclotomic subgroup of sixth degree extensions, Public Key Cryptography - PKC 2010, Lecture Notes in Computer Science 6056 (2010), 209-223. MR2660744

13. D. Harris, Simultaneous field divisions: an extension of Montgomery's trick,(2008), Available at http://eprint.iacr.org/2008/199.

14. K. Karabina, Double-exponentiation in factor-4 groups and its applications, Twelfth IMA International Conference on Cryptography and Coding, Lecture Notes in Computer Science 5921 (2009), 336-350. MR2775632

15. _ Factor-4 and 6 compression of cyclotomic subgroups of $\mathbb{F}_{2^{4 m}}^{*}$ and $\mathbb{F}_{3}^{*}$, Journal of Mathematical Cryptology 4 (2010), 1-42. MR2660332(2011i:94076)

16. Torus-based compression by factor 4 and 6 , Accepted for publication in IEEE Transactions on Information theory, DOI 10.1109/TIT.2012.2184846; Earlier version available at http://eprint.iacr.org/2010/525.

17. N. Koblitz and A. Menezes, Pairing-based cryptography at high security levels, Tenth IMA International Conference on Cryptography and Coding, Lecture Notes in Computer Science 3796 (2005), 13-36. MR2235246 (2007b:94235)

18. A. Lenstra and E. Verheul, The XTR public key system, Advances in Cryptology - CRYPTO 2000, Lecture Notes in Computer Science 1880 (2000), 1-19. MR.1850033 (2002k:94026)

19. C. Lim and H. Hwang, Fast implementation of elliptic curve arithmetic in $G F\left(p^{n}\right)$, Public Key Cryptography - PKC 2000, Lecture Notes in Computer Science 1751 (2000), 405-421. MR 1864790

20. P. Montgomery, Speeding the Pollard and elliptic curve methods of factorization, Mathematics of Computation 48 (1987), 243-264. MR866113 (88e:11130)

21. Y. Nogami, M. Akane, Yumi Sakemi, H. Kato, and Y. Morikawa, Integer variable $\chi$-based ate pairing, Pairing-Based Cryptography - Pairing 2008, Lecture Notes in Computer Science 5209 (2008), 178-191. MR2733913

22. K. Rubin and A. Silverberg, Torus-based cryptography, Advances in Cryptology - CRYPTO 2003, Lecture Notes in Computer Science 2729 (2003), 349-365. MR2093203 (2005e:94202)

23. Compression in finite fields and torus-based cryptography, SIAM Journal on Computing 37 (2008), 1401-1428. MR2386274 (2009d:94101)

24. M. Scott, Implementing cryptographic pairings, Pairing-Based Cryptography - Pairing 2007, Lecture Notes in Computer Science 4575 (2007), 177-196. MR2423639 (2009e:94081)

25. M. Shirase, D. Han, Y. Hibin, H. Kim, and T. Takagi, A more compact representation of XTR cryptosystem, IEICE Transactions on Fundamentals of Electronics, Communications and Computer Sciences E91-A (2008), 2843-2850. 
26. P. Smith and C. Skinner, A public-key cryptosystem and a digital signature system based on the Lucas function analogue to discrete logarithms, Advances in Cryptology - ASIACRYPT '94, Lecture Notes In Computer Science 917 (1994), 357-364.

27. M. Stam and A. Lenstra, Speeding up XTR, Advances in Cryptology - ASIACRYPT 2001, Lecture Notes in Computer Science 2248 (2001), 125-143. MR1934519 (2003h:94049)

28. __ Efficient subgroup exponentiation in quadratic and sixth degree extensions, Cryptographic Hardware and Embedded Systems - CHES 20022523 (2003), 159-174.

29. M. van Dijk, R. Granger, D. Page, K. Rubin, A. Silverberg, M. Stam, and D. Woodruff, Practical cryptography in high dimensional tori, Advances in Cryptology - EUROCRYPT 2005, Lecture Notes in Computer Science 3494 (2005), 234-250. MR.2352191 (2008h:94053)

30. M. van Dijk and D. Woodruff, Asymptotically optimal communication for torus-based cryptography, Advances in Cryptology - CRYPTO 2004, Lecture Notes in Computer Science 3152 (2004), 151-178. MR2147501 (2007b:94224)

Department of Combinatorics and Optimization, University of Waterloo, Waterloo, Ontario, Canada N2L 3G1

E-mail address: kkarabin@uwaterloo.ca 